\title{
Mécanismes économiques expliquant les bas salaires dans les services à la personne
}

Une analyse centrée sur les aides à domicile

Economic Mechanisms Explaining Low Wages in Personal Services. An Analysis

Focused on Home Helpers

\section{François-Xavier Devetter et Emmanuelle Puissant}

\section{(2) OpenEdition}

\section{Journals}

\section{Édition électronique}

URL : http://journals.openedition.org/travailemploi/8826

DOI : 10.4000/travailemploi.8826

ISSN : 1775-416X

\section{Éditeur}

DARES - Ministère du Travail

\section{Édition imprimée}

Date de publication : 1 juillet 2018

Pagination : 31-64

ISSN : 0224-4365

\section{Référence électronique}

François-Xavier Devetter et Emmanuelle Puissant, « Mécanismes économiques expliquant les bas salaires dans les services à la personne », Travail et Emploi [En ligne], 155-156 | Juillet-décembre 2018, mis en ligne le 11 juillet 2019, consulté le 10 octobre 2019. URL : http://journals.openedition.org/ travailemploi/8826 ; DOI : 10.4000/travailemploi.8826 


\title{
Mécanismes économiques expliquant les bas salaires dans les services à la personne
}

\author{
Une analyse centrée sur les aides à domicile
}

François-Xavier Devetter ${ }^{*}$ Emmanuelle Puissant ${ }^{* *}$

\begin{abstract}
Cet article a pour objectif de montrer comment les faibles salaires dans les services d'aide à domicile, dont les emplois sont considérés comme «peu qualifiés », résultent d'un ensemble de mécanismes qui sont le fruit d'une construction sociopolitique et socioéconomique. Ces derniers relèvent de stratégies publiques comme privées, que nous cherchons à clarifier à partir d'une synthèse de travaux empiriques portant sur le champ des services à la personne. Trois mécanismes de non-reconnaissance de ces métiers sont identifiés (nier ou réduire les « qualités » mobilisées, construire une offre abondante et enfin diviser la main-d'œuvre), chacun d'entre eux se déclinant à la fois sur le plan des orientations politiques nationales et des stratégies des ressources humaines de la part des employeurs. Le secteur de l'aide à domicile apparaît comme illustratif de tendances à l'œuvre dans bien d'autres activités de service fortement féminisées (nettoyage, hôtellerie-restauration ou commerce).
\end{abstract}

$\mathrm{L}$ es mutations de l'emploi depuis trente ans ont été caractérisées par la croissance des inégalités des revenus et des qualifications. Le développement d'une « économie de la connaissance » s'est certes traduit par la croissance d'emplois très qualifiés (cadres et ingénieurs) mais il s'est également accompagné d'un maintien voire d'un renouveau d'emplois peu qualifiés (MÉDA, VENNAT, 2004). Ce phénomène de polarisation des emplois a été étudié tant aux États-Unis qu'en Europe (JoLly, 2015), à la fois sous l'angle des transformations en termes de qualité des emplois (FERNANDEZMacias, 2012) et en termes de qualifications (OEsCH, MEnEs, 2011). Il a également

\footnotetext{
* Université Lille, Centre lillois d'études et de recherches sociologiques et économiques (Clersé) ; Lille Douai ; francois-xavier.devetter@imt-lille-douai.fr.

** Université Grenoble Alpes, Centre de recherche en économie de Grenoble (Creg) ; emmanuelle.puissant@univgrenoble-alpes.fr.
} 
été étudié plus précisément en France (Ast, 2015). Pour autant, ces travaux s'appuient souvent sur les transformations technologiques qui ont, notamment, touché l'industrie. À quelques exceptions près (DWYER, 2013), les spécificités des métiers féminins, majoritairement dans les services, ne sont pas au cœur de l'analyse de la polarisation.

C'est précisément sur un segment de ce marché du travail dit « non qualifié », dans les services où les femmes sont fortement représentées, que cet article se concentre : le segment des activités de services à la personne ${ }^{1}$ (SAP), et plus spécifiquement les emplois d'aide à domicile ${ }^{2}$ (encadré 1). Comme d'autres activités identifiées à partir de l'enquête Conditions de travail réalisée par la Direction de l'animation de la recherche, des études et des statistiques (Dares) en 2013, comme les nettoyeurs, serveurs et aides de cuisine, vendeurs en habillement, agents de service, etc., il s'agit de segments particulièrement dégradés du marché du travail, où se cumulent notamment précarité, faible rémunération, conditions de travail souvent pénibles, absence de formation et de perspectives de promotion (CAROLI, GaUtiÉ, 2009 ; CARrÉ, Tilly, 2012 ; KALLEBERG, 2011). Loin d'être en voie de régression, ce segment du marché du travail s'est plutôt développé et regroupe des emplois pour lesquels les perspectives de recrutement demeurent importantes (ABOUBADRA et al., 2014) sans que le niveau des salaires associés s'améliore réellement.

Bien que la catégorie statistique des employés non qualifiés ne soit pas pleinement stabilisée (Burnod, Chenu, 2001 ; Amossé, Chardon, 2006 ; Rose, 2012), il semble que de nombreux emplois de services appartiennent à ce type de catégorie, dont la position en bas de l'échelle est traditionnellement expliquée par trois mécanismes qui s'auto-entretiennent. D'abord, la faible productivité des emplois ne justifie qu'une rémunération basse (CAHUC, 2001). Le salaire minimum interprofessionnel de croissance (Smic) peut même apparaître comme supérieur au niveau de salaire que leur productivité marginale devrait impliquer, ce qui justifierait alors des subventions spécifiques $^{3}$ (BLANCHET, RAVALET, 1995). Ensuite, les compétences nécessaires étant

\footnotetext{
1. Les activités d'aide à domicile sont les activités réalisées au domicile privé des personnes dites fragiles (personnes âgées, personnes handicapées, familles considérées comme étant en difficulté). Ces activités, reconnues comme appartenant aux activités sociales et médico-sociales par la loi du 2 janvier 2002 rénovant l'action sociale et médicosociale, sont englobées dans le champ des services à la personne depuis le plan Borloo de cohésion sociale de 2005. Ce champ des SAP est beaucoup plus large et intègre les employées de maison, les activités de bricolage, d'assistance informatique, ou encore de jardinage à domicile. L'aide à domicile auprès des personnes dépendantes représente plus de $57 \%$ de l'activité des organismes de SAP, l'entretien de la maison $26,3 \%$, le jardinage-bricolage et la garde d'enfants environ $5 \%$ chacun (KulanthaIVElu, 2018). La majorité des salariées aides à domicile n'ont pas de diplôme en lien avec le secteur et même le diplôme d'État d'auxiliaire de vie sociale (DEAVS) demeure rare (TRABUT, 2014). 2. Cet article se concentrera sur le secteur de l'aide à domicile, au sein duquel les métiers, les formations et les diplômes se sont historiquement construits. Le champ des services à la personne sera lui aussi abordé, dans la mesure où la création du marché des services à la personne relève d'une stratégie publique de légitimation des bas salaires dans l'ensemble des activités qui en relèvent.

3. Parallèlement, le niveau du Smic relativement élevé (en termes absolus et de ratio Smic/salaire médian) peut amener les employeurs à « compenser » en intensifiant le travail et/ou en investissant moins dans les dimensions non salariales de la qualité de l'emploi. Ève CAROLI et Jérôme GAUTIÉ (2009) expliquent ainsi le paradoxe français des travailleurs à bas salaires relativement bien payés par rapport à leurs homologues d'autres pays européens, mais plus mécontents de leur salaire car soumis à des conditions de travail plus dures selon les enquêtes européennes.
} 


\section{ENCADRÉ 1}

\section{De l'aide à domicile aux services à la personne : l'évolution d'un secteur sous la pression des politiques publiques}

Si, depuis le plan Borloo de 2005, les services à la personne regroupent un ensemble d'activités hétéroclites, relevant d'histoires, de logiques et de régulations très différentes (DEVETTER et al., 2015), les activités d'aide à domicile (AD) connaissent quant à elles une certaine cohérence tant historique, statistique, professionnelle que législative. L'aide à domicile est ici définie à partir de ses conventions collectives comme l'ensemble des services s'adressant à un public fragile (personnes âgées ou handicapées, familles en difficulté) et rendus au domicile privé des bénéficiaires. Les premières formes d'entreprises d'aide à domicile, à la fin des années 1940, ont été associatives. Les premières conventions collectives datent de 1970 et se développent surtout au cours des années 1980, dans le double sens où elles se multiplient et où elles sont de plus en plus détaillées. Par la suite, elles sont complétées par des accords de branche, notamment au cours des années 1990. Durant les années 2000, les trois conventions collectives du secteur privé sans but lucratif fusionnent, et une nouvelle convention collective, celle des entreprises lucratives de services à la personne, voit le jour.

Depuis 1996, et avec une nette accélération à partir de 2005, le secteur a été ouvert à la concurrence, de sorte qu'il se caractérise aujourd'hui par la coexistence de deux modalités d'emploi et quatre types d'employeurs (schéma, infra). Cette évolution s'inscrit dans un processus amorcé dès les années 1980, visant au développement des emplois familiaux comme levier de réduction du chômage par la création d'emplois de proximité, réputés accessibles sans qualification particulière. Ainsi, les politiques de l'emploi et les politiques d'exonérations sociales et fiscales se sont fortement développées sur ce champ.

largement présentes dans la population, l'offre de travail potentielle est abondante, ce qui tire le salaire de marché vers le bas, notamment en période de chômage élevé. Enfin, dans une approche en termes de relations industrielles, l'activité étant souvent dispersée, ces métiers bénéficient d'une moindre organisation collective et d'un plus faible taux de syndicalisation que dans les autres secteurs (Western, Healy, 1999).

Ces trois mécanismes renvoient à des constats empiriques largement documentés. Ces processus socioéconomiques concourent à naturaliser la situation des salariées de l'aide à domicile et légitiment ainsi le maintien des bas salaires. La faible productivité des emplois, l'existence d'une offre de travail abondante ou encore la dispersion de la main-d'œuvre ne sont pas des données émergeant spontanément mais sont au contraire la conséquence de politiques publiques et de stratégies de gestion des ressources humaines plus ou moins intentionnelles. Celles-ci ont fait l'objet d'analyses approfondies à propos des emplois industriels qui ont mis en évidence des mécanismes de 
déqualification ${ }^{4}$ touchant les ouvriers de métier (BRAVERMAN, 1976 ; HAAKKESTAD, FRIDBERG, 2017), l'existence d'une « armée de réserve industrielle » (MARX, 1867), ou encore les mécanismes de division de la main-d'œuvre (MARGLIN, 1974 ; GORDON et al., 1982 ; PERRAUDIN et al., 2006).

L'objet de cet article, qui s'inscrit dans une approche socioéconomique, est de mettre au jour, à partir d'une synthèse de travaux empiriques portant sur le champ des services à la personne, et principalement sur l'aide à domicile, les processus correspondant à ces mécanismes économiques, tant dans leur contenu que dans leur maintien voire leur développement, expliquant ainsi le maintien de niveaux de salaires faibles. Le premier mécanisme (la faible rémunération) consiste à nier ou à réduire les « qualités » mobilisées par les salariées dans leur travail quotidien. Cette réduction a ici une double signification : leur sous-estimation, mais aussi une déqualification (BRAVERMAN, 1976), c'est-à-dire la suppression de ces qualités, notamment par des outils d'organisation du travail. L'article se concentrera prioritairement sur la première signification. Le deuxième mécanisme porte sur la construction d'une offre abondante, dont l'objectif est de maintenir un déséquilibre entre offre et demande au profit de la demande de travail, c'est-à-dire des employeurs. Enfin, le troisième mécanisme identifié consiste à diviser la main-d'œuvre, en accentuant la concurrence et en affaiblissant les collectifs de travail, pour en diminuer les capacités d'expression (le tableau 2, p. 53, synthétise ces différents éléments). Notre analyse prend principalement appui sur nos études empiriques antérieures, sur les métiers de l'aide à domicile et ponctuellement sur d'autres métiers au bas de l'échelle des rémunérations, lorsque les mécanismes identifiés se retrouvent de manière structurante. Cette démarche permet de souligner la vertu heuristique du secteur de l'aide à domicile pour comprendre le fonctionnement de segments dégradés du marché de l'emploi (encadré 2).

\section{ENCADRÉ 2}

\section{Méthodologie utilisée}

Cette contribution a un objectif d'ordre théorique : elle vise à synthétiser les processus concourant à la non-reconnaissance salariale et symbolique des métiers de l'aide à domicile. Elle s'appuie ainsi sur dix études empiriques réalisées entre 2008 et 2017 sur ce champ. Il s'agit principalement de matériaux concernant le secteur de l'aide à domicile, mais le secteur du nettoyage ainsi que les métiers de la petite enfance y sont également abordés. Au total, ce sont 442 entretiens semi-directifs menés auprès des salariés, des employeurs et des régulateurs de ces champs d'activité (liste des études et des entretiens réalisés en annexe). Les éclairages ponctuels sur d'autres secteurs seront mobilisés quand les mécanismes identifiés y ont également été observés.

4. Par « déqualification » nous entendons ici un double processus de sous-estimation et de non-reconnaissance des compétences mises en œuvre d'une part, et de suppression des compétences mobilisées par le développement d'outils ou de formes d'organisation du travail réduisant l'autonomie des professionnelles d'autre part. 


\section{Négation des compétences et des pénibilités}

Au cœur des théories d'inspirations néo-classiques visant à expliquer les écarts de salaire, figurent la théorie du capital humain et la théorie hédonique des salaires. Dans le premier cas, les différences de rémunérations compensent les coûts directs et indirects d'acquisition du capital humain et sont expliquées par les différences de productivité (approchées par les différences de formations ou de qualifications) tandis que, dans le second cas, ce sont les pénibilités subies qui servent à justifier les écarts de salaires.

Or la faiblesse des rétributions ne tient pas tant à des niveaux faibles de ces deux caractéristiques de l'emploi (qualification et pénibilité) qu'à leur non-reconnaissance sociale. En effet, faire le lien entre ces deux dimensions de l'emploi et leurs compensations requiert que les niveaux de pénibilité comme de qualification soient bien établis. Cette valorisation sociale n'émerge pas spontanément, elle dépend de la volonté des acteurs concernés. Or, dans le champ des services à la personne, l'absence de valorisation est en jeu, mais également, en amont, la reconnaissance de l'existence même de compétences mobilisées ou de pénibilités subies.

\section{La naturalisation des compétences}

La négation des compétences a été étudiée de manière approfondie par les travaux sociologiques ou économiques relatifs aux métiers féminins, tant dans l'industrie (Guilbert, 1966 ; Maruani, Nicole-Drancourt, 1989) que dans les services (Dussuet, 1997 ; GAdRey et al., 2004 ; 2009 ; Folbre, 2001 ; Budig et al., 2002).

Les mécanismes mis en évidence dans ces travaux alimentent une première logique, qui consiste à ne pas considérer les compétences requises comme identifiables ni objectivables. Elles sont présentées comme «naturelles », caractéristiques des « savoir-être » des personnes. Surtout, n'étant pas formalisées, elles ne peuvent pas constituer une « barrière à l'entrée ». Les compétences et qualités mobilisées sont alors considérées comme largement partagées parce qu'elles sont en grande partie acquises dans la sphère privée et informelle, dans le cadre d'une socialisation marquée par le poids du genre (de la force des hommes à la minutie des femmes). De ce fait, les compétences requises ne sont pas transmises dans des écoles de formation particulières, ce qui constitue un frein à leur objectivation et visibilisation. Un autre processus favorisant le partage large de ces savoir-faire supposés non spécifiques repose sur des dispositifs techniques ou organisationnels qui réduisent le travail à une succession de gestes ou de postures simples. Ce processus a été particulièrement bien étudié dans le cadre de la mise en œuvre d'une organisation scientifique du travail - dans une logique proche de celle décrite par Harry BRAVERMAN (1976) à propos de l'industrie - dans les services les plus taylorisés comme la restauration rapide (NKOUATCHET, 2005). Il émerge plus récemment dans les services, plus « relationnels », comme dans les services à la personne, et s'appuie sur le développement d'une qualité certifiée, de procédures et mécanismes de standardisation associés à un contrôle renforcé de la main-d'œuvre, ou 
encore d'une dévalorisation de certaines compétences «profanes » (CRESSON, 2006). Les conflits autour de la reconnaissance des compétences et de leur certification sont intenses mais, parallèlement, des mécanismes de disqualification explicites peuvent être observés. Deux exemples permettent d'illustrer ces processus : les modalités de recrutement des assistantes maternelles d'une part et les choix opérés par les politiques publiques et les employeurs vis-à-vis des aides à domicile d'autre part.

La situation des assistantes maternelles est particulièrement emblématique. La structuration progressive de la profession et la croissance de la régulation publique (Tirmarche-IsSEMAnN, 2011) n'ont pas été accompagnées d'une promotion de la formation (initiale ou continue) qui, « si elle est jugée comme intéressante voire utile, est rarement perçue comme nécessaire et encore moins indispensable » (ABALLÉA, 2005, p. 60). Par exemple, l'exigence posée de s'inscrire au module obligatoire « Prise en charge de l'enfant au domicile » du certificat d'aptitude professionnelle (CAP) petite enfance n'implique pas l'obligation d'en valider les épreuves. Les enquêtes qualitatives font ressortir les contradictions des autorités publiques comme celles des parents employeurs, qui oscillent ainsi en permanence entre la reconnaissance du « travail remarquable » effectué par ces salariées et l'insistance mise sur l'accessibilité de cette profession à tous, sans barrière à l'entrée, au nom de la nécessaire lutte contre le chômage. Cette dissonance entre deux objectifs contraires, valorisation du « métier de mère » et volonté d'encadrement professionnalisé, produit une situation où les processus de recrutements s'apparentent à un « tri social » renvoyant aux caractéristiques sociodémographiques des salariées (lieu et cadre de l'habitation, apparences, etc. ; cf. VOZARI, 2014) dont les critères sont trop implicites pour être légitimés. Le refus de reconnaître socialement les qualités mobilisées peut être en partie expliqué par le fait que la collectivité n'accepte que de payer peu ces professionnelles (un tiers du Smic horaire pour un enfant pris en charge). Jeanne FAGNANI et Antoine MatH (2012) ont montré, sur la base d'analyses économétriques, que globalement les parents employeurs n'étaient pas disposés à verser un salaire plus élevé en cas de qualifications accrues. La quasi-absence de valorisation salariale de l'ancienneté dans la convention collective les concernant vient conforter cette réalité.

Dans le cas de l'aide à domicile, les politiques publiques se sont positionnées en faveur de la professionnalisation des emplois et des métiers. Mais cette volonté a été à la fois tardive et ambivalente (PUISSANT, 2011). Tardive, car pour l'aide à domicile aux personnes âgées, le premier diplôme national (le certificat d'aptitude à la formation d'aide à domicile) date de 1990 tandis que le premier diplôme d'État du secteur (diplôme d'État d'auxiliaire de vie sociale, DEAVS) date de 2002, alors que le certificat d'aptitude à la fonction d'aide-soignant (Cafas), par exemple, existe depuis 1956. L'attitude des pouvoirs publics a été ambivalente car si ce diplôme d'État constitue une avancée considérable dans la reconnaissance du caractère qualifié de certains métiers du secteur ${ }^{5}$, sa dimension

5. La création et la diffusion du DEAVS depuis 2002 se sont d'ailleurs accompagnées d'une croissance sensible des niveaux de rémunération moyens des aides à domicile bien que ces derniers demeurent toujours très bas (DEVETTER et al., 2017). 
facultative pour exercer le travail d'aide à domicile en réduit fortement la portée, aussi bien symbolique que matérielle (Dussuet, Puissant, 2015). Plus encore, ce diplôme n'est reconnu que par un type d'employeurs (associatif). Il n'est pas pris en compte dans la classification des autres conventions collectives (celles des particuliers employeurs, des entreprises à but lucratif et centres communaux et intercommunaux d'action sociale). Le caractère ambivalent de cette professionnalisation, au sens de reconnaissance des qualifications, résulte sans doute de la tentative de concilier deux représentations sociales relatives au vieillissement, qui débouchent sur des objectifs de politiques publiques sensiblement différents : l'une concerne la nécessité de développer des emplois qualifiés dans le secteur médico-social pour s'occuper des personnes âgées, et l'autre est relative à la volonté de contenir le coût public que représente la prise en charge de la dépendance.

Les employeurs et les politiques de ressources humaines $(\mathrm{RH})$ mises en place dans les structures d'aide à domicile jouent également un rôle dans le processus de naturalisation des compétences. Les entretiens réalisés avec des employeurs montrent que les critères de recrutement utilisés portent souvent sur des caractéristiques liées au sexe des candidates, sur leur statut extra-professionnel (de fille et de mère notamment), voire sur la « disponibilité temporelle » dont elles doivent faire preuve, mais bien plus rarement sur les qualifications qu'elles ont pu acquérir (DEVETTER, RoussEAU, 2009). De même, l'accès à la formation des salariées est relativement modeste dans ce secteur et demeure très dépendant du statut des employeurs : très restreint pour les salariées de particuliers employeurs, un peu moins pour celles des structures prestataires (tableau 1). Toutefois, la part des formations non qualifiantes reste prédominante comme le rappelle la directrice des ressources humaines (DRH) d'une association :

«Le plus important dans les formations, c'est que les salariées se rendent compte de la complexité de ce qu'on leur demande [...]. Vous incitez les salariées à passer le DEAVS ? Ah non, non ! Si elles veulent, pas de problème bien sûr, la VAE [validation des acquis de l'expérience] est un droit ! Mais on ne le demande pas, ce n'est pas forcément nécessaire. Et puis, si toutes les salariées qui interviennent chez des personnes malades étaient diplômées, ce ne serait pas possible avec notre tarif horaire ! [rires] »

(Entretien avec une directrice RH d'une association en Rhône-Alpes, 2011

[Lamotte, Puissant, 2013])

Cette vision est d'ailleurs assez fréquemment intégrée par les salariées ellesmêmes, qui vont même jusqu'à ne pas déclarer avoir de diplôme :

« Je savais que si je leur disais [le fait d'avoir le DEAVS], ils ne m'auraient pas prise, j' aurais coûté trop cher. C'était arrivé à une collègue, donc je n'ai pas voulu prendre le risque. »

(Prise de parole d'une salariée d'une association dans la Drôme, lors d'une rencontre autour du vieillissement de la population, 2008 [LAMOTTE, Puissant, 2010])

Les employeurs perçoivent ainsi souvent le diplôme comme impliquant un coût salarial supplémentaire et un risque de départ (vers les emplois en structures d'hébergement type établissement d'hébergement pour personnes âgées dépendantes [Ehpad] 
par exemple) plus que comme une opportunité d'améliorer la qualité du travail et du service. Ceci peut contribuer à expliquer la faiblesse des formations dans les entreprises et associations et leur quasi-absence dans l'emploi direct (tableau 1).

TABLEAU 1 - Les formations dans les services à la personne

\begin{tabular}{lccc} 
& Secteur public & $\begin{array}{c}\text { Particulier } \\
\text { employeur }\end{array}$ \\
\hline & $\begin{array}{c}\text { Structure } \\
\text { prestataire privée } \\
\text { (association ou } \\
\text { entreprise) }\end{array}$ & & 0,5 \\
\hline $\begin{array}{l}\text { Suivi d'une formation formelle durant les quatre } \\
\text { semaines précédant l'enquête (FORMEL) }\end{array}$ & 2 & 12 & 1 \\
$\begin{array}{l}\text { Suivi d'un cours ou d'un stage durant les trois } \\
\text { derniers mois (COURSTAG) }\end{array}$ & 10 & 2,5 & 0,5 \\
$\begin{array}{l}\text { Participation à des cours, conférences ou } \\
\text { séminaires au cours des trois derniers mois } \\
\text { (SEMCONF) }\end{array}$ & 1 & 62 & 5 \\
$\begin{array}{l}\text { Cette formation a été suivie au moins partiellement } \\
\text { durant le temps de travail (LWORHA, si la } \\
\text { personne suit ou a suivi une formation) }\end{array}$ & 53 & & \\
\hline
\end{tabular}

Champ : aides à domicile et employées de maison en emploi $(\mathrm{N}=6522)$.

Source : enquête Emploi 2015.

\section{Des pénibilités niées et sans contrepartie}

Ces formes d'invisibilisation des compétences se retrouvent également en matière de pénibilités et de conditions de travail. En effet, les cadres institutionnels de connaissance et reconnaissance des conditions de travail et d'emploi ont été construits dans le contexte de la « société salariale » (CASTEL, 1995), plutôt industrielle et masculine. Or, la tertiarisation et la féminisation du travail et de l'emploi sont des défis importants en termes de renouvellement de ces cadres analytiques. Ainsi, si les pénibilités dites « industrielles » sont aujourd'hui plutôt bien référencées et détaillées dans les nomenclatures statistiques, les conventions collectives et les accords de branche, il n'en va pas de même pour les pénibilités dites tertiaires, liées à la « relation de service », selon le sens que leur donne Jean GADREY (2003). Des travaux récents ont toutefois essayé de caractériser ces formes de pénibilité (Devetter, Messaoudi, 2013) et les risques professionnels associés (DUSSUET, 2013).

L'aide à domicile figure ainsi parmi les secteurs fortement exposés aux accidents du travail et aux maladies professionnelles, avec plus de 47 jours d'arrêts pour un million d'heures travaillées contre 33 en moyenne pour l'ensemble des salariés (CNAMTS, 2016). La pénibilité tient notamment à la multiplicité des déplacements au cours d'une même journée, entre deux interventions, comme en témoignent les deux extraits d'entretien suivants :

« On a un quart d'heure pour arriver chez les personnes. Donc aujourd'hui, mettons, je travaille sur mon secteur. J'fais mettons une demi-heure là-bas et après je cours sur 
l'autre côté. Et donc aujourd'hui, ils nous arrangent plus comme avant. Donc avant on avait, on avait une personne. Des fois je fais une personne d'un côté du secteur et je retourne après là-bas et après je retourne de l'autre côté. Voilà, et donc tu cours... »

(Aide à domicile, femme, 2016, [DEVETTER et al., 2016])

«- Le week-end, c'est que des demi-heures, c'est pour des repas en fait. [...] Ça m'arrive de faire 9 h 30 quand même sur une journée.

Q : - Et vous avez des interventions de deux heures ?

- Ah j'ai presque jamais, y a pas... Le maximum, c'est une heure. Et à part le lundi après-midi chez cette dame, j'ai de 2 h 15 à 2 h 45 . C'est quand on va faire les courses. »

(Aide à domicile, femme, 2016, [DeVETTER et al., 2016])

Or la régulation publique du secteur et son mode de financement (par le paiement à l'heure de l'usager via un tarif qui demeure relativement bas) ne favorisent pas une meilleure prise en compte de ces risques professionnels. Ils ne permettent pas, par exemple, d'intégrer correctement les temps considérés comme « non productifs » (inter-vacations notamment), ce qui contribue à intensifier le travail et à rendre plus difficiles les conditions de son exercice. De même, la médecine du travail ne joue pour les aides à domicile qu'un rôle partiel. Elle ne couvre tout d'abord qu'une part réduite des salariées (les employées des particuliers y échappent totalement). Ensuite, pour les salariées qu'elle couvre, elle ne peut pas accéder au lieu de travail (domicile privé) et il en va de même pour l'inspection du travail. Elle intervient enfin souvent tardivement (peu de politiques de prévention) et de manière très complexe à gérer pour les structures : face aux difficultés de santé rencontrées par les salariées, le médecin du travail peut recommander des mises en « incapacité partielle » (ne pas porter des charges supérieures à $5 \mathrm{~kg}$ par exemple) ; or ces décisions médicales sont de fait incompatibles avec l'exercice du métier, et sont régulièrement critiquées par les employeurs que nous avons pu interroger. L'organisation du travail et les politiques RH ont pour effet de relativiser les pénibilités. Des travaux sur les associations en général (HÉLY, 2009), et sur celles d'aide à domicile en particulier (Dussuet, 2005 ; PUISSANT, 2010), ont mis en exergue la prédominance du service rendu aux usagers au détriment des conditions de travail. Nos enquêtes montrent que l'organisation du travail peut être, de plus, un élément d'aggravation des pénibilités : la non-prise en compte de celles-ci peut conduire les employeurs à mettre en place des outils d'organisation du travail et de politique RH augmentant les cadences de travail :

«On fait pour le mieux, c'est tout, mais souvent nous sommes dépassées : avec leur demi-heure, on ne peut pas tout faire, ce n'est pas possible. Non non. On ne peut pas. Dans la demi-heure, il faut qu' on assume le repas, qu'on assume les choses. Souvent, s'il manque des choses, il faut aller vite courir acheter, quand c'est pas loin, c'est bon, mais quand c'est plus loin, on ne peut pas. Et en plus dès que le temps arrive, qu'est-ce qu'on fait des gens? »

(Aide à domicile, femme, 2016, [DEVETTER et al., 2016]) 
Ainsi, le morcellement du travail est facteur de pénibilité et d'augmentation des risques professionnels : le risque d'accidents de la route notamment est multiplié, de même que, plus globalement, celui de dégradation de l'état de santé des salariées :

«Mais il n'était pas permanent, le stress, il était après sur la fin, le stress, c'était le temps, les distances que j'devais parcourir à vélo. Les distances, parce que moi j'avais dix interventions dans une journée et c'était voilà. Pas l'temps d'manger, pas l'temps de rentrer manger l'midi hein... »

(Aide à domicile, femme, licenciée pour inaptitude suite à un accident de vélo survenu entre deux interventions [DEVETTER et al., 2016])

La tendance à l' augmentation du nombre d'interventions de moins d'une heure au domicile des personnes âgées accroît ce morcellement, lequel peut être encore renforcé par la diffusion de modalités de contrôle des horaires via des outils de télégestion (Dussuet et al., 2017).

Ce processus est aussi à l'œuvre pour d'autres professions, comme les salariés du nettoyage (et notamment les femmes), qui souffrent d'une invisibilisation des pénibilités qu'elles subissent. Karen Messing et ses co-auteures $(1993,1998)$ analysent ainsi de manière approfondie comment les tâches considérées comme moins difficiles sur le plan physique (dénommées « travaux légers ») impliquent souvent des contraintes tout aussi voire plus intenses que les travaux initialement perçus par les employeurs et les donneurs d'ordres comme plus exigeants (travaux dits « lourds ») : la réalité des conditions de travail s'écarte ainsi nettement de leur caractérisation ex ante par l'encadrement. De même, l'exposition à des substances chimiques demeure largement invisible dans ces métiers où les produits toxiques (sprays divers, eau de javel, anticalcaire, etc.) semblent d'une grande banalité, alors que leurs conséquences sur la santé sont désormais bien établies par les travaux scientifiques (Zock, 2005 par exemple).

Ces secteurs sont même ceux où la sinistralité est la plus élevée en matière d'accidents du travail et de maladies professionnelles : le nombre d'accidents par million d'heures de travail atteint ainsi 52 dans le nettoyage courant des bâtiments et 75 dans l'aide à domicile, contre une moyenne de 23 pour l'ensemble des salariés couverts par le régime général ou de 39 dans le secteur du bâtiment et des travaux public (CNAMTS, 2016). Pourtant, l'analyse des données statistiques relatives aux métiers de l'aide à domicile et du nettoyage témoigne d'un décalage important entre la déclaration de certaines pénibilités (le rythme de travail perçu est ainsi plutôt faible) et l'état de santé des personnes. Le lien entre les conditions de travail et l'état de santé est mal évalué, les salariées semblant sous-déclarer les pénibilités liées à leur travail. Plusieurs travaux ont mis en évidence l'importance du genre dans cette sous-estimation des pénibilités (GOLLAC, VOLKOFF, 2006), qui peuvent contribuer à des situations de travail dégradées et non compensées sur le marché du travail : si les salariées ressentent faiblement les pénibilités et ne les expriment pas d'une manière collective, ces emplois n'ont pas à être compensés, notamment par un salaire supérieur au salaire minimum. Les salariées sont alors amenées à gérer individuellement leur capacité de résistance : le recours 
généralisé au temps partiel apparaît comme un moyen de «tenir» dans un travail dont la pénibilité demeure forte. Ce type de stratégies existe également dans d'autres secteurs d'activité : c'est notamment le cas des ouvrières agricoles qui optent pour un emploi en contrats à durée déterminée (CDD) successifs, afin d'avoir des pauses entre deux contrats (Roux, 2018).

\section{Construction d'un excès d'offre de travail}

Les logiques que nous avons décrites précédemment légitiment l'assertion selon laquelle il n'y a pas de nécessité d'une qualification spécifique ni, par conséquent, d'une rémunération pour celle-ci. Ce faisant, ces logiques suppriment les éventuelles « barrières » à l'entrée de certaines professions et facilitent l'émergence de dispositifs conduisant à développer l'offre de travail (sans avoir à recourir aux canaux classiques de développement des formations et de croissance des rémunérations) car la perception sociale du service rendu (caractérisé par un consentement à payer très faible) empêche d'attribuer au travail qui sous-tend ces services un salaire de marché supérieur au salaire minimal. Sans valeur marchande, mais socialement nécessaire, ce travail doit être offert par une main-d'œuvre « suscitée ». Il convient alors d'orienter suffisamment de personnes vers ces métiers et de «mettre au travail » ceux, et souvent celles, qui « devraient » accepter ces emplois. Pour comprendre ce phénomène, deux voies principales sont ici évoquées, en distinguant pour chacune ce qui relève des politiques publiques et des stratégies des employeurs : il s'agit, d'une part, de mettre au travail des catégories spécifiques de main-d'œuvre et, d'autre part, de subventionner (à la fois du côté de l'offre de travail et du côté de la demande concernée par ces services) ce travail « peu productif ».

\section{La mise au travail de catégories « sous-mobilisées » : mères de famille et immigrées}

La première façon d'accroître l'offre de travail est de susciter l'activité de certaines catégories perçues comme « sous-employées ». L'exploitation de gisements de maind'œuvre s'impose dès qu'un volume important d'emplois vacants est identifié car, en l'absence d'une nouvelle offre de travail, des tensions sur les recrutements pourraient se manifester et pousser les salaires à la hausse. Il est de ce point de vue assez paradoxal de constater l'ampleur des témoignages qui, dans les médias, mentionnent un « secteur en tensions » en même temps que l'absence d'amélioration des conditions d'emploi ou de salaire y prévalant. La réalité des difficultés de recrutement est de fait pour le moins complexe à établir (ESTRADE, 2013), notamment parce que de nombreuses entreprises ont des pratiques de recrutements « extensives » : ainsi, quantité d'annonces « permanentes » sont déposées, qui visent à attirer des candidatures pour des postes... qui ne seront ouverts que si de nouveaux clients se présentent. Les entretiens auprès 
des employeurs soulignent souvent à la fois l'importance des flux de candidatures ${ }^{6}$ et la difficulté à fidéliser ces salariées. Les éventuelles tensions sur les recrutements ne s'expliquent ainsi pas par une faiblesse quantitative de l'offre de travail mais plutôt par un décalage entre les exigences des employeurs et la qualité des emplois proposés. Pour y faire face, les employeurs et les politiques publiques ne visent pas l'amélioration (jugée trop coûteuse) de la qualité de l'emploi mais cherche plutôt à accroître le plus possible le vivier potentiel de recrutement.

La faiblesse du taux d'emploi (et non plus l'importance du taux de chômage) est alors mise en exergue (ZAJDELA, 2009), notamment dans le cas des jeunes de moins de 25 ans, des femmes immigrées ou encore des mères de famille. Des mesures spécifiques sont mises en œuvre pour encourager l'accès de ces catégories au marché du travail. Ces mesures peuvent viser à réduire des obstacles réels ou supposés empêchant l'expression d'une offre de travail en proposant des aides à la mobilité (aides à l'obtention du permis de conduire par exemple) ou la création de contrats de travail adaptés (contrats étudiants, contrats à temps partiel choisi, etc.). Elles se traduisent également par l'adaptation des modes de recrutements, tant en termes de critères de sélection (avec l'accent mis sur des caractéristiques sociodémographiques spécifiques) qu'en termes de mises en relation (forums, speed dating, annonces de postes permanentes, etc. ${ }^{7}$ ). Le rôle d'orientation des intermédiaires sur le marché du travail est ici également central : Pôle emploi, mission locale, services sociaux, etc., participent directement à la construction d'un flux de demandeuses d'emploi vers ces métiers. De manière plus analytique, on observe que l'existence d'une main-d'œuvre déqualifiée (i.e. dont la qualification s'est étiolée - ou est considérée comme telle par les employeurs - notamment suite à une suspension de l'activité professionnelle), "mère en reprise d'activité », a été renforcée par des dispositifs publics comme le congé parental dont les effets en matière de qualification et de carrière féminine ont été largement mis en évidence (MÉDA, PÉRIVIER, 2007).

Cette situation est directement observable dans le secteur des services à la personne. En effet, les intermédiaires du marché du travail, notamment Pôle emploi et les services d'insertion des conseils départementaux, sont directement incités à orienter les demandeuses d'emploi vers ces métiers (Alberola et al., 2011). Une aide à domicile rencontrée confirme cette orientation quasi systématique vers les services à la personne :

«Quand j'ai eu besoin de travailler pour avoir un salaire, la seule chose qu'on me proposait, c'était travailler avec les personnes âgées. J'ai pas hésité longtemps, de toute façon, y avait pas le choix. »

(Aide à domicile, femme, 2016 [DEVETTER et al., 2016])

\footnotetext{
6. « On reçoit des CV tous les jours. Tous les jours, des dames passent à l'agence. Tous les jours. » (Employeuse, entreprise de SAP, 2018).

7. Dans le cas de notre étude sur l'externalisation de l'entretien de dix-neuf collèges, deux salariées de l'entreprise prestataire ont par exemple expliqué avoir été recrutées « en cinq minutes, devant la grille ». Le responsable de secteur qui a procédé à l'embauche confirmera que « dans ce secteur [géographique], c'est pas difficile de trouver vu le taux de chômage » (Responsable de secteur, homme, 2015 [DEVETTER, STEINAUER, 2017]).
} 
Dans la même lignée que les acteurs publics, les employeurs cherchent également à attirer certains segments de la main-d'œuvre en insistant sur les compétences « naturelles » nécessaires mais également en annonçant tenir compte des contraintes spécifiques des « mères de famille ». L'entreprise $\mathrm{O} 2$ publie ainsi des témoignages de salariées sur sa page « recrutement » qui, toutes, insistent sur la compatibilité de leurs horaires avec les contraintes familiales. La sélection du personnel peut emprunter des voies complexes telles que la segmentation des profils en fonction des offres de temps de travail spécifiques. Certains employeurs proposent explicitement des emplois à temps très réduits offrant un complément de salaire faible mais compatible avec des charges familiales, comme l'illustre le propos suivant :

«Le second profil ? La mère de famille qui a sacrifié sa carrière pour élever ses enfants et qui souhaite un complément de revenu et des horaires cohérents avec ses obligations (pas de mercredi, etc.). Elles travaillent vingt heures par semaine. Tout le monde est content. »

(Chef d'entreprise, homme, 2008 [Devetter, Rousseau, 2009])

La population immigrée (et notamment les femmes) est une seconde catégorie de main-d'œuvre orientée vers les services à la personne. Si les politiques d'importation de main-d'œuvre ont été étudiées dans le cas des emplois industriels ou de la construction (JounIN, 2009), elles sont moins directement mises en avant dans le cas des emplois peu qualifiés des services. Pourtant, il s'agit d'une longue tradition, visible dans les services domestiques, où le recours à une main-d'œuvre allogène est récurrent : bretonne et belge au début du Xx ${ }^{\mathrm{e}}$ siècle, antillaise dans les années 1930, espagnole puis portugaise dans les années 1960-1970, etc. (MARTIN-HUAN, 1997). Les politiques publiques ont récemment relancé ce processus, symbolisé par un accord entre l'Agence nationale des services à la personne et le ministère de l'Immigration en 2008 visant à orienter les primo-arrivants prioritairement vers ces services (DEveTTER et al., 2015). De même, un rapport du CENTRE D'ANALYSE STRATÉGIQUE intitulé « Besoins de main-d'œuvre et politique migratoire » (mai 2006, p. 53) affirme que c'est dans les métiers de la santé et des services personnels et domestiques « qu'un recours, au moins temporaire, à une immigration plus importante peut se justifier ». Du côté des employeurs et des branches professionnelles, les stratégies sont assez explicites lorsque les recrutements s'appuient sur les réseaux « ethniques » (ANDERSON, 2000 ; BAKAN, STASILIUS, 2005 ; IBOS, 2013 ; AvRIL, 2014). L'embauche prioritaire de salariées immigrées passe ensuite par d'autres formes de naturalisation des compétences ; lors d'un entretien en 2009, un membre d'une organisation patronale soulignait «l'amour des personnes âgées des femmes africaines ». Ici, la naturalisation est à comprendre comme un processus social considérant des compétences ou des qualités comme innées, niant ainsi qu'elles résultent de processus de socialisation ancrés dans des rapports sociaux de sexe et d'origine (Dussuet, 2010).

Ainsi, pour ne pas avoir à entrer en concurrence sur le marché du travail, les employeurs des services à la personne (mais également du nettoyage ou de la restauration) cherchent à exploiter des segments « sous-utilisés » de la main-d'œuvre. Ils 
peuvent en ce sens jouer un rôle d'insertion dans l'emploi des catégories de salariées qui en sont traditionnellement éloignées mais à des niveaux de qualité de l'emploi particulièrement faibles.

Au-delà du secteur des services à la personne, les autres métiers des emplois dégradés des services sont également concernés par des mécanismes de ce type. Le cas le plus significatif est probablement celui des salariés de l'hôtellerie-restauration : les « catégories spécifiques » (immigrés et étudiants notamment) y jouent un rôle central. Comme le rappellent Pierre BARRON et ses co-auteur.e.s (2011), citant notamment André Daguin, président de l'Union des métiers de l'industrie hôtelière, le recrutement de salariés sans-papiers apparaît comme " une nécessité économique ». De même, loin d'être une main-d'œuvre d'appoint, les jeunes en études constituent une part conséquente des salariés et contribuent à maintenir une offre de travail peu exigeante (NKOUATCHET, 2005).

\section{Rendre le travail «payant»}

Développer une main-d'œuvre disponible passe certes par le recours à des populations « nouvelles » mais nécessite également de convaincre les salariées d'accepter les emplois en question. À nouveau, tant les politiques publiques ${ }^{8}$ que les employeurs participent généralement à cet objectif soit en cherchant à rendre le travail « payant » (c'est-à-dire plus rémunérateur et profitable à l'individu qui l'occupe), soit en réduisant la capacité des futur.e.s salarié.e.s à refuser des emplois peu attractifs (CATTACIN et al., 2002 ; FAGAN et al., 2006).

La transformation du dispositif du revenu minimum d'insertion (RMI) en revenu de solidarité active (RSA) dont l'objectif est de faciliter le cumul partiel de revenus d'activité et d'aides sociales, est probablement l'exemple le plus important des mesures publiques allant dans cette logique de workfare («faire que le travail paye »). La politique publique en faveur des services à la personne insistait ainsi sur la nécessité d'activer le RSA en faveur de ce champ ${ }^{9}$ et l'Agence nationale des services à la personne (ANSP) tout comme les entreprises ${ }^{10}$ communiquaient abondamment sur la possibilité de s'appuyer sur ce dispositif. Sur son site internet, l'ANSP informait clairement sur la possibilité pour les salariées de bénéficier du RSA ${ }^{11}$ et terminait

\footnotetext{
8. Les aides publiques présentées dans ce paragraphe concernent d'abord les subventions directes à l'offre de travail mais elles peuvent également prendre la forme de subventions à la demande qui abaissent le « coût » du travail tout en soutenant indirectement le salaire net versé aux salarié.e.s.

9. Ainsi, selon Michèle Debonneull (2008, p. 31), il faut encourager le RSA « de façon à permettre aux entreprises d'avoir moins de difficultés à trouver des candidats puisqu'elles pourraient proposer à tous ceux qui recherchent un travail à temps plein d'être payés comme s'ils travaillaient à temps plein alors qu'ils travailleraient à temps partiel ». 10. Pour un exemple parmi de nombreux autres, voir le communiqué « Adecco à domicile et l'Afpa forment des bénéficiaires du RSA » : http://www.groupe-adecco.fr/articles/adecco-a-domicile-et-lafpa-forment-des-beneficiaires-du-rsa, consulté le 5 décembre 2018.

11. Page « Bénéficiez du RSA ! » de l'ANSP : https://archives.entreprises.gouv.fr/2013/www.servicesalapersonne. gouv.fr/beneficiez-du-rsa-pour-ameliorer-votre-quotidien-(12164)d41d.html, consulté le 5 décembre 2018.
} 
par le slogan «le RSA, ça sert à ça !». Cette agence a d'ailleurs soutenu l'envoi d'une brochure d'information sur le RSA à l'ensemble des salarié.e.s rémunéré·e.s en chèques emploi service universel (Cesu).

Les mesures visant à favoriser une offre dite « raisonnable » d'emploi ou cherchant à conditionner les aides sociales à une offre de « travail » prolongent cette logique en France comme plus largement en Europe. Les emplois non qualifiés dans des champs proches des services à la personne constituent ainsi de gros contingents de mini-jobs en Allemagne (HIPP et al., 2015). De même, les nombreuses subventions et exonérations sociales et fiscales dont bénéficient les emplois peu qualifiés et, plus encore, le secteur des services à la personne participent à la possibilité de payer un travail considéré comme ne valant pas ce qu'il coûte (i.e. où la productivité perçue est considérée comme inférieure au salaire versé) (DEvetTER, JANY-CATRICE, 2010).

Au niveau des employeurs, cette logique passe par des stratégies de recrutement ciblant spécifiquement des salariées «peu employables » sur les autres segments du marché du travail. Ainsi, un employeur (DEVETTER, Rousseau, 2009) insiste en 2008 sur les critères de recrutement permettant d'éviter un taux de rotation trop élevé : « Les filles jeunes ne viennent pas chez nous pour rester. C'est un métier de passage. Mais pour des dames de 40 ans, elles ne se voient pas faire autre chose. »

Le fait que les salariées aient connu un «passage difficile » peut être également un critère important :

« On est dans une région sinistrée avec le textile. On retrouve beaucoup de gens qui viennent de ce secteur, qui ont entre 35 et 50 ans. C'est là où on a le plus de facilités à recruter car les gens ont un professionnalisme, un respect du client, un respect de l'employeur qui est autrement plus dimensionné que les plus jeunes. »

(Chef d'entreprise, 2008 [Devetter, Rousseau, 2009])

Ces modalités de sélection peuvent expliquer le paradoxe apparent entre une très faible employabilité des salariées selon les critères traditionnels (qualification, mobilité, etc.) et leur grande facilité à être embauchées : ce sont justement des critères habituellement négatifs qui sont ici recherchés par certains employeurs (DEVETTER, Rousseau, 2009). Ainsi, il arrive qu' un employeur explique qu'il distingue les salariées « véhiculées » et « non véhiculées » et privilégie, dès que c'est matériellement possible, les secondes. L'impact parfois négatif de la détention du diplôme DEAVS décrit dans la précédente section relève indirectement de ce même processus d'indifférenciation qui alimente une offre abondante. Ainsi, la faible employabilité des salariées est à comprendre non pas comme menant à des difficultés à être recrutées, mais comme des caractéristiques en termes de faible mobilité géographique et professionnelle notamment, menant à des positions dégradées sur le marché du travail.

En dehors du champ des services à la personne, le recours à des contrats aidés peut illustrer également cette stratégie visant à rendre le travail à la fois payant (en complétant le salaire par des aides sociales) et difficilement refusable, car proposé par les services d'accompagnement social. Le cas des agent·e·s de service des collectivités 
locales (incluant depuis 2005 celles-ceux des établissements scolaires) en est un exemple emblématique. Dans le cadre de monographies réalisées sur dix-neuf collèges (DevetTER, SteinAuer, 2017), il est apparu que le renfort de main-d'œuvre que constituent les salarié.e.s en contrats aidés n'était en rien un supplément mais bien un moyen de compléter des équipes nettement insuffisantes. Selon l'enquête Conditions de travail, les emplois aidés représentent ainsi plus de $12 \%$ des postes d'agent.e.s de service des établissements scolaires (profession 525A).

\section{Segmentation et division de la main-d'œuvre}

Enfin, un troisième mécanisme peut bloquer ou inverser le processus de construction d'une voie de professionnalisation et d'accroissement des rémunérations : le maintien d'une division de la main-d'œuvre, qui freine les possibilités de constitution d'un collectif de travail homogène et limite la portée des revendications syndicales. On identifie ici deux processus participant à la division de la main-d'œuvre : le recours à une grande variété de types d'employeurs et de statuts (pour effectuer le même métier) ou la mise en concurrence de différents métiers (pour effectuer les mêmes tâches) d'une part, et les mesures faisant obstacle à la structuration d'un collectif de travail, d'autre part. À nouveau, chacun de ces deux processus est relayé par les politiques publiques et par les politiques RH développées par les employeurs.

\section{La concurrence entre différents types d'employeurs, de métiers et de conventions collectives}

Parmi les dix métiers qui figurent en bas de la hiérarchie salariale, quasiment tous sont soumis à des modes d'organisation multiples, qui mettent en concurrence différents types d'employeurs : coexistence de statuts, de conventions collectives nationales $(\mathrm{CCN})$ ou encore de formes d'emplois spécifiques ( « extra » et CDD très courts dans la restauration, externalisation et sous-traitance dans l'hôtellerie et le nettoyage, etc.). La coexistence de structures publiques, de structures associatives et d'entreprises privées, voire d'emplois directs par un particulier employeur pour organiser des activités comparables est une situation relativement peu fréquente d'un point de vue général, mais très présente pour les employées de maison, les aides à domicile, les assistantes maternelles ou encore les agentes d'entretien. De même, plusieurs de ces métiers sont parmi les plus exposés à la croissance de la sous-traitance. Les agentes de nettoyage et les femmes de chambres sont particulièrement exposées à l'externalisation : $60 \%$ des nettoyeuses relèvent de la branche de la propreté, tandis que les autres sont salariées d'entreprises conservant leurs agentes d'entretien en interne mais dépendant de branches diverses. Or, le recours à la sous-traitance se traduit par une dégradation nette des conditions d'emploi pour les salariées, tant en France (Abasabanye et al., 2016 ; Devetter, Steinauer, 2018) qu'à l'étranger (Holley, RainNie, 2012 ; Grimshaw et al., 2014). 
Le secteur des services à la personne est emblématique de cet éclatement entre différents types d'employeurs et de modalités d'emploi, qui débouche sur une grande hétérogénéité de règles applicables (notamment issues des $\mathrm{CCN}$ ). Loin d'avoir cherché à unifier le secteur, les politiques publiques ont favorisé la diversification de ces modalités d'emploi depuis les années 1980, avec les premières mesures d'incitation au développement de l'emploi direct. Puis, dès les années 1990 mais avec une nette accélération en 2005 avec le plan de développement des services à la personne, les incitations en faveur des entreprises lucratives se sont renforcées, accentuant la concurrence entre les types d'employeurs, les différences de statut ayant de fortes répercussions en termes de conditions de travail et d'emploi (LeFEBVRe, 2012 ; DeVETTER et al., 2017).

Cette mise en concurrence des types d'employeurs est aussi de leur fait, que ce soit au niveau des branches ou des structures elles-mêmes. La pluralité des conventions collectives $^{12}$, qui est caractéristique de la branche de l'aide à domicile, est un facteur déterminant de la concurrence par les prix entre différents types d'employeurs et d'offreurs de services. Elle concourt de plus à un éclatement et à un affaiblissement de la représentation du personnel, d'autant plus que les centrales syndicales peinent à uniformiser leur modalité de représentation : les négociateurs des différentes branches appartiennent le plus souvent à des fédérations distinctes ( « santé social ${ }^{13} »$ pour la branche de l'aide à domicile et « services commerce ${ }^{14} »$ pour les entreprises lucratives de services à la personne). La mise en concurrence relève aussi des stratégies des structures elles-mêmes, via la démultiplication des agences ou des unités employeuses permettant, consciemment ou non, de contourner les seuils de représentation du personnel ou d'optimiser certains dispositifs fiscaux (PERRAUdin et al., 2006). La pratique des franchises pour les entreprises à but lucratif ou l'éclatement des associations en petites structures locales en sont des exemples flagrants. Un autre exemple de stratégies d'employeurs exacerbant la concurrence entre ces différents types de services est le recours à deux modalités d'emploi : la prestation et le mandatement (schéma). Les prestataires sont des organisations collectives qui peuvent avoir différents statuts (public ou privé, à but lucratif ou non) : elles emploient des aides à domicile, les paient et déterminent leur planning, etc. Les salariées travaillent ainsi chez des usagers qui financent un service d'aide, mais ne les emploient ni ne les rémunèrent directement. Dans ce cas, les salariées relèvent de la convention collective correspondant au statut de leur employeur (CCN de 2013 du prestataire lucratif des services à la personne, ou CCN de 2010 du prestataire associatif). Mais nombre de ces organisations développent en leur sein, à côté de leur service prestataire, un service dit mandataire. L'organisation n'emploie alors pas les salariées : ce sont les usagers qui les emploient directement et les rémunèrent, principalement en Cesu. Les salariées relèvent donc de la convention collective du particulier employeur. L'organisation mandataire est

12. Le rattachement à une convention collective dépend du statut juridique de l'employeur et non des activités effectuées.

13. Il s'agit plus précisément de la Fédération des organismes sociaux pour la CGT, de la Fédération « santé sociaux » pour la CFDT.

14. Il s'agit ici de la Fédération du commerce pour la CGT, et des services pour la CFDT. 
SCHÉMA - Les différentes modalités d'emploi dans les services à la personne

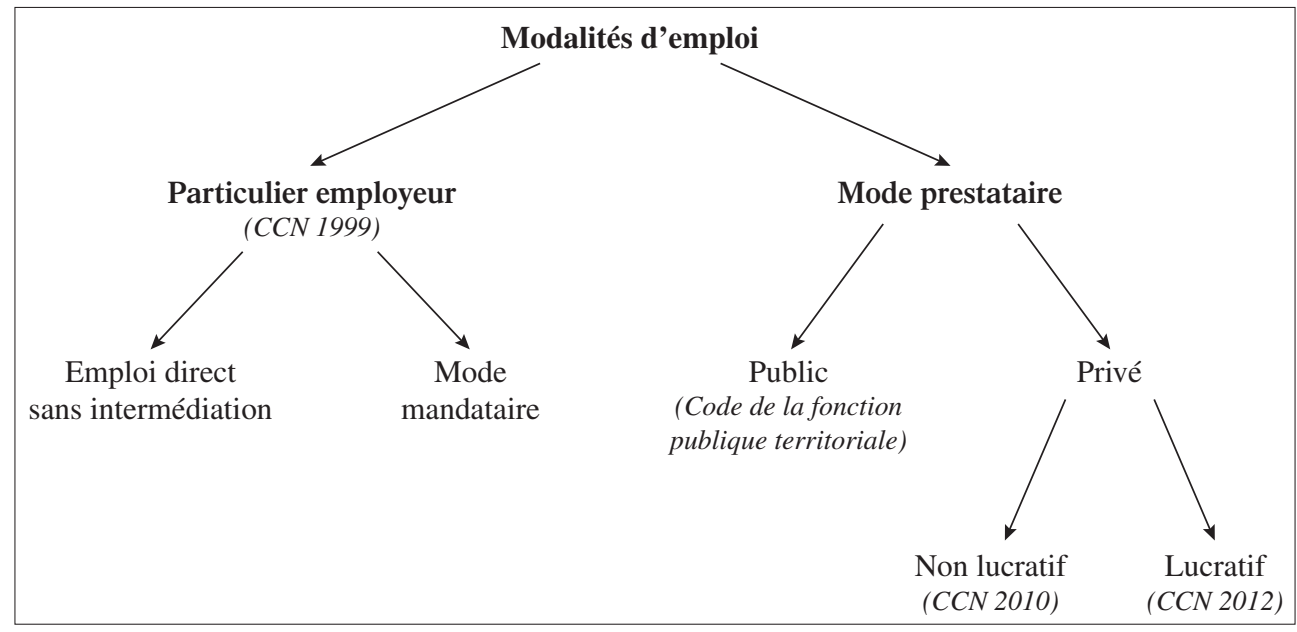

Source : auteurs.

payée par les usagers uniquement pour les aider dans la gestion administrative de l'emploi (établissement des fiches de paie, congés, arrêts maladie, etc.) et des plannings en cas d'absence, pour assurer par exemple un remplacement temporaire. Ce sont souvent les mêmes salariées qui, au cours de la même journée, peuvent être ainsi amenées à travailler sous plusieurs conventions collectives, parfois chez les mêmes usagers.

Cette situation constitue un frein important à la représentation syndicale, comme l'atteste un extrait d'entretien réalisé en 2012 avec l'animatrice du collectif régional Rhône-Alpes Confédération générale du travail (CGT) « aide à domicile » (LAMOTTE, Puissant, 2013) :

«Les services à la personne, c'est un vrai défi syndical ! Il faut que les fédérations se parlent, les filles ne se reconnaissent pas forcément de l'action sociale [sic], mais plus de la santé. Et puis les associations sont souvent tellement petites, les filles sont isolées. On réfléchit à la création d'une union économique et sociale, pour que les employeurs soient obligés de se regrouper, et qu'on puisse créer des syndicats dans les associations. On réfléchit aussi à créer des syndicats de territoire, pour toutes les aides à domicile qui voudraient se syndiquer. Le syndicalisme doit évoluer pour répondre à ces transformations, et pour permettre à ces filles de se syndiquer et se défendre, mais ce n'est pas évident. »

La concurrence entre types d'employeurs peut être renforcée par une division entre des métiers initialement conçus comme distincts. Ainsi, historiquement, ce sont les activités les plus éloignées des tâches ménagères, réalisées par les aides à domicile, qui ont été reconnues comme requérant des compétences particulières. Le premier diplôme du secteur date des années 1950 ; il concerne l'aide à domicile auprès des familles et porte sur les activités dites «éducatives » auprès des enfants. Dans les services auprès des personnes âgées, cette reconnaissance a été plus tardive (années 1990) et a porté sur 
les activités d' « aide aux actes essentiels de la vie » (accord de branche du 29 mars 2002 sur les classifications des emplois), c'est-à-dire l'aide à la personne et non au foyer (aide à la mobilité, à la toilette, aux repas, etc.). La reconnaissance professionnelle s'opère donc de façon fragmentée : reconnaître le travail et les compétences des unes (aide à la personne, c'est-à-dire aide pour les activités éducatives auprès des enfants, et aide aux actes dits essentiels de la vie quotidienne, selon les termes employés dans la convention collective de la branche aide à domicile, c'est-à-dire aide à la toilette, à la mobilité, au repas, etc., pour les personnes âgées ou handicapées) revient implicitement à ne pas reconnaître le travail des autres (ARBORIO, 2012). La reconnaissance professionnelle s'effectue de fait par l'objectivation des seules tâches entrant dans les référentiels de l'action sociale et médico-sociale (qui relèvent de l'aide à la personne). Ainsi, pour les salariées n'effectuant pas ce type de tâches sur les personnes, mais effectuant principalement des tâches d'aide à l'entretien du logement (aide au ménage, repassage), les leviers de reconnaissance professionnelle n'existent pas. Cette division repose sur l'idée qu'exercer des activités, même principalement ménagères, chez des publics fragiles ne requiert pas de compétences professionnelles particulières, puisqu'aucun diplôme n'existe en la matière. On aurait tout à fait pu imaginer un autre processus, se concentrant sur les usagers : intervenir auprès d'usagers fragiles nécessite des compétences professionnelles particulières et donc des qualifications, puisque les tâches ménagères sont d'abord les supports d'une relation plus complexe et non une finalité en elles-mêmes (JANY-CATRICE, 2010). On retrouve cette tension dans la représentation des métiers dans l'extrait d'entretien suivant :

«Souvent, les familles des personnes nous prennent pour des femmes de ménage. Les personnes, elles, elles savent, elles voient bien qu'on est plus, qu'on est là pour elles, et pas seulement pour le ménage. Mais les familles, c'est pas pareil. Elles sont pas souvent là, elles pensent qu'on est des femmes de ménage. Alors qu'on est là pour leurs parents, quand ils sont déprimés, c'est à nous qu'ils parlent. Même des fois, les mamies, elles préfèrent me parler à moi qu'à leurs filles, elles se sentent plus libres, je sais pas...»

(Aide à domicile, 2012 [LAmotte, Puissant, 2013])

Cette division de la reconnaissance professionnelle peut être renforcée par l'organisation du travail dans certaines structures ; elle engendre alors une forte concurrence entre salariées. Le fait que des compétences soient reconnues et formalisées dans le cadre d'un diplôme, mais sans que ce diplôme soit une barrière à l'entrée du secteur, a conduit à une qualification différenciée des aides à domicile ${ }^{15}$. Seule l'obtention du diplôme d'État, et non plus l'ancienneté, permet le passage à la catégorie qualifiée. Si

15. La convention collective de la branche de l'aide à domicile (secteur privé non lucratif) de 2012 reprend l'accord de branche du 29 mars 2002 qui définissait quatre catégories d'aides à domicile : 1) A : agente à domicile, pas de qualification, 2) B : employée à domicile, en cours de validation des acquis ou BEP-BTS (brevet d'études professionnelles-brevet de technicien supérieur), 3) C : auxiliaire de vie sociale : titulaire du diplôme d'État d'auxiliaire de vie sociale - qui n'existe plus depuis le $1^{\mathrm{er}}$ janvier 2016, 4) D : technicienne d'intervention sociale et familiale pour l'aide auprès des enfants dans les familles en difficultés. 
le DEAVS est accessible en validation des acquis de l'expérience (il a d'ailleurs été le premier diplôme, en 2002, à être accessible en VAE), nous avons montré dans des travaux antérieurs (PUISSANT, 2010) que dans un secteur composé de femmes ayant souvent eu un rapport compliqué avec l'école et les études, le fait de s'engager dans une démarche de VAE s'avère problématique pour beaucoup. La formalisation d'une pratique professionnelle peut être un exercice difficile pour bon nombre d'aides à domicile, ce qui explique qu'elles sont nombreuses à s' auto-censurer en refusant de s'engager dans une démarche de VAE, ou alors à ne pas valider l'intégralité des modules. Ainsi, une aide à domicile ayant plus de vingt ans d'ancienneté, mais sans qualification officielle, sera en catégorie A - non qualifiée. Certaines salariées parlent parfois de « trahison », interprétant cette catégorisation comme un moyen de ne pas reconnaître le travail qu'elles effectuent depuis longtemps, et pour lequel elles ont parfois suivi des modules de formation.

«Moi, ça fait plus de quinze ans que je fais ce métier. Je sais que je connais mon métier, je vais pas aller passer le diplôme. C'est pas eux qui vont décider si je connais mon métier. Ils ont qu'à aller voir les personnes chez qui je vais, ils sont contents. Moi, je sais que je connais mon métier. Et quand les filles elles passent leur diplôme, après, elles se sentent plus, elles veulent plus vider les poubelles ! Comme si c'était moi qui devais vider les poubelles à chaque fois ! »

(Aide à domicile, 2016 [DEVETTER et al., 2016])

Le ressentiment est d'autant plus vif que la catégorisation des aides à domicile n'engendre pas des différences concrètes dans le travail réalisé par les unes et les autres. Dans les faits, les employeurs manquent de main-d'œuvre qualifiée pour accompagner le vieillissement et l'accroissement des situations de handicap : des salariées non qualifiées continuent donc de travailler chez des usagers en situation de dépendance, comme le montre l'extrait suivant.

«Le week-end, on intervient que chez des personnes très dépendantes. Alors, si je suis censée ne faire que du ménage, pourquoi je travaille tout le temps le week-end ? Là, ça les dérange pas que j’ai pas le diplôme et que je sois payée au Smic pour aider à la toilette !»

(Aide à domicile, 2016 [DeveTTER et al., 2016])

Ces formes de glissements de tâches ou de concurrence entre professions partageant un espace de travail ne sont pas propres aux services à la personne et des phénomènes comparables s'observent dans l'hébergement social et médico-social (NIRELlo, 2015), et même à l'hôpital (ARBorio, 2012).

Ces formes d'éclatement se retrouvent également de manière croissante dans les services publics, à l'image des emplois d'entretien au sein des collèges. L'analyse de l'organisation du travail d'entretien au sein de dix-neuf collèges dans un département qui expérimente le recours à du personnel en sous-traitance (DEVETTER, STEINAUER, 2017) souligne que dans ces établissements, les activités de nettoyage peuvent être effectuées par des salariées relevant de quatre statuts différents : les plus stables sont titulaires de la fonction publique territoriale ; viennent ensuite des contractuelles embauchées sur des 
périodes de dix mois et demi, puis des salariées en contrats aidés et enfin des agentes de nettoyage salariées d'une société prestataire. Ces quatre formes d'emploi sont affectées à des tâches largement comparables, bien que les personnels concernés ne disposent ni des mêmes ressources (formation, expérience, insertion professionnelle ; équipement matériel ; etc.) ni des mêmes conditions d'emploi et de salaires. La rémunération mensuelle peut ainsi varier du simple au double selon les statuts (en raison de grilles salariales plus ou moins avantageuses), mais surtout en fonction des temps de travail octroyés pour effectuer le nettoyage d'une surface donnée (de 41 heures à 20 heures par semaine pour des surfaces équivalentes). Cet exemple montre que l'existence d'une pluralité de statuts pour effectuer une même activité (divisant et affaiblissant les capacités de négociation des salariés) n'est pas propre aux seuls services à la personne.

Ces professions de services partagent ainsi le fait d'être divisées entre une grande diversité de statuts ou de métiers, pourtant mobilisés pour effectuer les mêmes tâches et remplir les mêmes missions.

\section{L'affaiblissement des collectifs de travail}

À cette division «statutaire » s'ajoute une organisation du travail bien souvent éclatée, qui tend à isoler les salariées et qui empêche l'émergence d'un collectif de travail. Cette situation se retrouve principalement au niveau des structures d'emploi mais s'appuie également sur des régulations publiques peu favorables.

En effet, les métiers de services peu qualifiés ont été sensiblement influencés par les politiques de flexibilisation mises en œuvre depuis une vingtaine d'années : facilitation du travail à temps partiel, flexibilisation du temps de travail, développement du travail indépendant, etc. Ces dispositifs ne s'adressent pas spécifiquement aux emplois peu qualifiés des services. Mais ils ont été particulièrement mobilisés dans ce type d'emplois : ainsi, les auto-entrepreneurs sont en nombre important dans les services à la personne (ThiÉRUS, 2014), le secteur de la propreté (DENIS, 2018) ou les services de livraison.

Dans les services à la personne, les caractéristiques de l'activité et le fait qu'elle se déroule au domicile des bénéficiaires tend « naturellement » à isoler les travailleuses. Pour autant, des formes d'organisation allant à l'encontre de cet isolement, comme le modèle triangulaire - usager/salarié/association - inventé dans le cadre associatif (PuISSANT, 2010), ont permis la création d'espaces collectifs de travail, indispensables à la constitution d'un groupe professionnel (ARTOIS, 2015). Or, depuis les années 1990, et avec une nette accélération depuis le début des années 2000, une remise en cause de cette organisation triangulaire (où une structure employeur s'insère entre l'usager et la salariée) s'opère. Les politiques publiques l'ont de fait fortement encouragée, d'abord via les incitations faites en faveur de l'emploi direct, puis en favorisant des " prestataires » à but lucratif dont l'unique rôle est la mise en relation (seule la dimension juridique de la fonction employeur étant ici assurée). La création d'un marché des services à la personne en 2005 a en effet encore davantage fragilisé le mode prestataire, 
en facilitant la concurrence et la pression sur les coûts. Si, dans un premier temps, par des exonérations fiscales et sociales diverses, le plan Borloo a encouragé une croissance rapide des prestataires à but lucratif, cet élan a ensuite été freiné. L'étude d'un cabinet d'experts a alerté dès 2010 sur les fermetures des entreprises privées lucratives qui, sans exonérations importantes, étaient fortement déficitaires dès qu' elles s'implantaient en dehors des grandes métropoles (BELze, 2011).

Plusieurs travaux (VATAN, 2014 ; Le Roy, Puissant, 2016) ont depuis attiré l'attention sur le fait que le modèle économique de référence implicitement suivi par les pouvoirs publics (ou les conseils départementaux pour la prise en charge des personnes âgées), notamment en termes de tarification, correspondait à la situation de l'emploi direct. Plusieurs conseils départementaux utilisent ainsi l'indicateur du temps de travail dit «productif » pour établir leur tarification et le temps de travail qu'ils sont prêts à financer sur fonds publics. Or ce temps dit productif ne tient pas compte des temps de travail effectués en dehors du domicile des usagers, même quand ils sont constitutifs du service qui y est réalisé (PuISSANT, 2010 ; LEFEBVRE, 2012 ; Dussuet, 2013). Tous les temps qui constituent l'une des spécificités du modèle prestataire ont de ce fait tendance à être fortement réduits, n'étant plus financés. Un système collectif, avec des postes de coordination et d'encadrement intermédiaire, des temps de formation, des temps de réunion, de droits d'expression, etc., est nécessairement plus coûteux qu'un emploi direct, où sont seulement prises en compte les activités qui se déroulent au domicile privé des personnes. Cette concurrence incite de plus en plus les pouvoirs publics locaux à ne retenir dans les tarifs horaires, qu'ils fixent avec les structures, que les heures dites « productives » (c'est-à-dire les heures d'intervention au domicile qui représentent aux alentours de $80 \%$ du volume global de travail dans les structures prestataires ; AUBE-MARTIN et al., 2010).

Cette évolution se répercute sur l'organisation du travail et les politiques RH : la rationalisation observée depuis le milieu des années 2000 dans les associations s'accélère et se généralise à l'ensemble du mode prestataire. Cela se manifeste d'abord par la tendance à la remise en cause des « usages » dans les structures, c'est-à-dire les acquis issus de négociations locales, qui représentent des améliorations informelles par rapport à ce que prévoient les conventions collectives et accords de branche (temps de pause, reconnaissance des temps de trajet dépassant le quart d'heure, jours de récupération, primes de fin d'année, etc.). Cela se traduit ensuite par la remise en cause des espaces de travail collectifs : réduction des postes de coordination (leur nombre et leurs prérogatives), diminution des temps d'échanges avec les collègues et des temps de travail collectifs. Enfin, cela s'accompagne d'une généralisation des outils de contrôle, comme la télégestion, qui contribuent à augmenter les cadences de travail et à déqualifier le travail :

« Avec la télégestion, on ne reconnaît plus notre capacité de jugement ! Si on estimait avant qu'on devait rester quinze minutes de plus alors que ce n'était pas prévu chez une personne, eh ben maintenant, on ne peut plus, on est contrôlé ! »

(Aide à domicile, 2016 [DeVETTER et al., 2016]) 
Ces stratégies se retrouvent parfois de manière très explicite dans des politiques RH, qui visent un maintien plus ou moins délibéré de l'isolement des salariés :

« Pas de rassemblement. Je suis pour qu'elles soient séparées. C'est l'expérience qui parle avec tout ce qui est syndicat, comité d'entreprise et tout. Plus les gens sont séparés, moins ils parlent, moins ils critiquent le patron ou l'encadrement. Je continue à faire en sorte qu'elles soient éclatées et le moins possible ensemble. »

(Chef d'entreprise, 2008 [DEvetter, Rousseau, 2009])

Encore une fois, ces mécanismes, synthétisés dans le tableau 2, sont observables, au-delà des services à la personne, dans la plupart des emplois de service dégradés. Nous avons déjà évoqué le cas des agentes d'entretien polyvalentes, qui se trouvent éclatées entre plusieurs statuts (DEVETTER, STEINAUER, 2017). Elles sont également souvent l'objet de mesures d'organisation du travail ayant vocation à éviter l'émergence de collectifs de travail : dans le cadre de l'externalisation de leur activité, la consigne donnée par le conseil départemental est explicitement de disposer de locaux distincts (servant à l'entreposage du matériel et aux pauses) pour les salariées du privé

\section{TABLEAU 2 - Synthèse sur les mécanismes de non-reconnaissance de la qualification} dans l'aide à domicile

\begin{tabular}{|c|c|c|c|}
\hline \multirow{2}{*}{\multicolumn{2}{|c|}{$\begin{array}{c}\text { Mécanismes de non-reconnaissance } \\
\text { de la qualification }\end{array}$}} & \multicolumn{2}{|c|}{ Niveau de décision et mise en œuvre } \\
\hline & & Politiques publiques & $\begin{array}{c}\text { Politiques } \\
\text { des ressources humaines }\end{array}$ \\
\hline \multirow{2}{*}{$\begin{array}{l}\text { Négation } \\
\text { des qualités } \\
\text { mobilisées }\end{array}$} & $\begin{array}{l}\text { Naturalisation } \\
\text { des compétences et absence } \\
\text { de « barrières à l'entrée » }\end{array}$ & $\begin{array}{l}\text { Reconnaissance tardive et } \\
\text { ambivalente des qualifications }\end{array}$ & $\begin{array}{l}\text { Critères de recrutement } \\
\text { extérieurs à la sphère } \\
\text { professionnelle et formations } \\
\text { non diplômantes }\end{array}$ \\
\hline & $\begin{array}{l}\text { Euphémisation } \\
\text { des pénibilités }\end{array}$ & $\begin{array}{l}\text { Difficile prise en considération } \\
\text { des pénibilités ; calcul restrictif } \\
\text { des tarifs ; absence de politique } \\
\text { de prévention }\end{array}$ & $\begin{array}{l}\text { Relativisation de la question des } \\
\text { conditions de travail ; } \\
\text { calcul restrictif du temps de } \\
\text { travail. }\end{array}$ \\
\hline \multirow{2}{*}{$\begin{array}{l}\text { Construction } \\
\text { d'un excès } \\
\text { d'offre }\end{array}$} & $\begin{array}{l}\text { Mise au travail des « mères », } \\
\text { des immigrées }\end{array}$ & $\begin{array}{l}\text { Orientation spécifique par les } \\
\text { intermédiaires de l'emploi }\end{array}$ & $\begin{array}{l}\text { Emploi des « mamans »; } \\
\text { utilisation des réseaux } \\
\text { « ethniques » }\end{array}$ \\
\hline & $\begin{array}{l}\text { Rendre le travail sous-payé } \\
\text { acceptable }\end{array}$ & $\begin{array}{l}\text { Revenu de solidarité active } \\
\text { (RSA) et prime pour l'emploi } \\
\text { (PPE); workfare : « faire que le } \\
\text { travail paye » }\end{array}$ & $\begin{array}{l}\text { Sélection des « inemployables » } \\
\text { ou des salariées d'appoint... }\end{array}$ \\
\hline \multirow{3}{*}{$\begin{array}{l}\text { Division de la } \\
\text { main-d'œuvre }\end{array}$} & $\begin{array}{l}\text { Concurrence entre } \\
\text { employeurs }\end{array}$ & $\begin{array}{l}\text { Incitation à la pluralité des } \\
\text { employeurs et des conditions } \\
\text { salariales }\end{array}$ & $\begin{array}{l}\text { Pluralité des conventions } \\
\text { collectives ; démultiplication des } \\
\text { agences ; recours au mandataire }\end{array}$ \\
\hline & $\begin{array}{l}\text { Concurrence entre } \\
\text { «métiers » }\end{array}$ & $\begin{array}{l}\text { Division de la reconnaissance } \\
\text { professionnelle }\end{array}$ & $\begin{array}{l}\text { Organisation du travail qui } \\
\text { exacerbe la concurrence au } \\
\text { quotidien entre qualifiées et non } \\
\text { qualifiées }\end{array}$ \\
\hline & $\begin{array}{l}\text { Affaiblissement des collectifs } \\
\text { de travail }\end{array}$ & $\begin{array}{l}\text { Emploi direct ; pressions de } \\
\text { la concurrence ; tarification } \\
\text { restrictive }\end{array}$ & $\begin{array}{l}\text { Remise en cause des temps } \\
\text { collectifs ; rationalisation } \\
\text { dans l'organisation du travail } \\
\text { augmentation des cadences }\end{array}$ \\
\hline
\end{tabular}

Source : auteurs. 
et pour les agentes du public ; l'organisation de cette sous-traitance établit sciemment deux catégories distinctes de personnels, invités à se penser en opposition, entre un « eux » et un « nous » clairement définis.

Alors que les niveaux de sortie de diplômées de l'enseignement secondaire et supérieur n'ont cessé de croître rapidement durant les années 1990 et 2000, la dynamique de qualification des emplois a connu des mouvements plus ambigus : croissance certes des emplois très qualifiés (notamment de statut cadre) mais reprise également de la création d'emplois considérés comme peu ou non qualifiés. Les politiques publiques visant à alléger le coût du travail ne sont pas étrangères à ce phénomène (GAUTIÉ, Margolis, 2009 ; Jaehrling, Mehaut, 2013), mais le rôle des politiques publiques et des stratégies de gestion des ressources humaines menées par les employeurs va cependant bien au-delà de ce seul soutien économique et encourage la polarisation des emplois tirée par la croissance des emplois de services dégradés, au moins autant que par la baisse des emplois industriels de niveau de qualification intermédiaire.

Ainsi, le maintien de nombreux emplois de services dans les catégories des « non qualifiés » résulte de plusieurs mécanismes qui se renforcent mutuellement : négation des qualités mobilisées et des pénibilités subies ; orientation d'un flux de main-d'œuvre fragile et « bon marché » vers ces segments ; stratégies de division de la main-d'œuvre rendant de facto impossibles l'action collective et les revendications susceptibles de l'accompagner. À partir de l'analyse de ces trois mécanismes qui ont des répercussions à la fois en termes d'invisibilisation et de déqualification, nous avons montré que le segment secondaire du marché du travail n'est pas, comme parfois présenté plus ou moins explicitement dans les approches de la segmentation du marché du travail (DOERINGER, PIORE, 1971 ; MARSDEN, 1999), un marché « non organisé » ou une forme proche du marché de concurrence pure et parfaite. Il est au contraire le fruit d'une construction sociale et politique.

Si nous avons mis en évidence le fait qu'un certain nombre de mécanismes de non-reconnaissance des compétences et même de déqualification du travail existent également dans d'autres segments de l'emploi dit secondaire, nous pouvons aller plus loin dans la compréhension de la dynamique des segments de métiers peu ou non qualifiés de services. En effet, le secteur de l'aide à domicile, et plus globalement le champ des services à la personne, a une vertu heuristique forte pour comprendre les freins réels à la qualification de pans entiers de métiers, dans lesquels les femmes sont surreprésentées. La facilitation de l'emploi direct (puis du recours à des prestataires très flexibles) et les pressions budgétaires sur l'emploi prestataire ont sans doute participé d'un laboratoire de déréglementation de la relation salariale, comme l'atteste la diffusion rapide de statuts permettant l'augmentation et la diversification des situations de travail se réalisant en dehors de tout cadre collectif de travail, en lien notamment avec le développement des plateformes numériques d'emploi. 
Cette évolution rappelle les processus décrits et analysés finement par les socioéconomistes radicaux américains à propos des emplois industriels (MARGLIN, 1974 ; BRAVERMAN, 1976 ; GORDON et al., 1982, par exemple). Le développement de certains services marchand(isé)s a nécessité la constitution d'une catégorie sociale (féminine et précaire), comme l'industrialisation au XIX ${ }^{\mathrm{e}}$ et au début du XX ${ }^{\mathrm{e}}$ siècle s'était appuyée sur la création d'une classe laborieuse bon marché.

Deux différences importantes existent toutefois, qui distinguent la situation actuelle de la période de l'industrialisation des siècles passés. La première concerne la trajectoire suivie, sur le plan individuel comme collectif : l'entrée de la main-d'œuvre féminine considérée comme peu qualifiée (DAUNE-RICHARD, 2003 ; DWYER, 2013) sur le marché du travail s'inscrit aussi dans un processus dialectique de réduction (partielle) de l'exploitation du travail féminin par les hommes dans le cadre de la société patriarcale (DelPhy, 2015). Ainsi, comme le salariat peut, dans une première phase, apparaître comme un progrès face au servage, le précariat féminin peut être considéré comme une première étape dans la reconnaissance de la valeur du travail féminin. La seconde différence renvoie à une interprétation en termes de freins durables à la reconnaissance des métiers dans les services : les activités concernées par l'intitulé « employé non qualifié » sont très largement relationnelles et consistent en des interactions sociales où la différence entre travail et service est très faible, contrairement à l'industrie où la machine et l'organisation du travail sont des médiations importantes entre le consommateur et le salarié. Cette partie relationnelle a des incidences sur la qualité du résultat plus que sur son « volume ». L'existence de gains de productivité (au sens industriel du terme) est plus difficilement identifiable et la faiblesse de ces gains « à partager » rend alors le conflit plus âpre et renforce l'enjeu, pour ceux qui en profitent, de ne pas voir reconnue la qualité - et donc la qualification - des services rendus et du travail effectué. La construction d'un compromis social dans ces services relationnels ne peut alors que passer par la croissance du « consentement à payer » exprimé par les consommateurs et plus encore par la collectivité. 


\section{BibLIOGRAPHIE}

AbaLLÉA F. (2005), «La professionnalisation inachevée des assistantes maternelles », Recherches et prévisions, $\mathrm{n}^{\circ}$ 80, pp. 55-65.

Abasabanye P., Bailly F., Devetter F.-X. (2016), "Does Contact Between Employees and Service Recipients Lead to Socially More Responsible Behaviours? The Case of Cleaning", Journal of Business Ethics, vol. 153, $\mathrm{n}^{\circ} 3$, pp. 13-824.

Aboubadra S., Argouarc'h J., Bessière S., Colin J.-F., Jolly C., Lainé F. (2014), Les Métiers en 2022. Résultats et enseignements, Rapport du groupe Prospective des métiers et qualifications, Paris, Dares, France stratégie.

Alberola E., Gilles L., Tith F. (2011), «Les services à la personne : un levier d'insertion pour les publics éloignés de l'emploi ? », Cahier de recherche, n $^{\circ} 288$, Crédoc.

Amossé T., ChARDON O. (2006), « Les travailleurs non qualifiés : une nouvelle classe sociale ? », Économie et statistique, $\mathrm{n}^{\circ}$ 393-394, pp. 203-229.

Anderson B. (2000), Doing the Dirty Work? The Global Politics of Domestic Labour, New York, Zed Books.

ARBorio A.-M. (2012), Un personnel invisible. Les aides-soignantes à l'hôpital, $2^{\mathrm{e}}$ éd. augmentée d'une préface de l'auteure, Paris, Économica, Anthropos.

ARTois P. (2015), La Pluralité des modalités de professionnalisation contemporaines : le groupe professionnel des aides familiales au ccur de tensions, Thèse de doctorat en sociologie, Bruxelles, Université libre de Bruxelles.

Ast D. (2015), « En 30 ans, forte progression de l'emploi dans les métiers qualifiés et dans certains métiers peu qualifiés de services », Dares analyses, $\mathrm{n}^{\circ} 028$.

Aube-Martin P., Bruant-Buisson A., Reboul (DE) J.-B. (2010), Mission relative aux questions de tarification et de solvabilisation des services d'aide à domicile en direction des publics fragiles, Rapport, Paris, Inspection générale des finances, Inspection générale des affaires sociales.

Avril C. (2014), Les Aides à domicile. Un autre monde populaire, Paris, La Dispute.

Bakan A. B, Stasilius D. K. (2005), Negotiating Citizenship: Migrant Women in Canada and the Global System, Toronto, University of Toronto Press.

BARron P., Bory A., TOurette L. (2011), On Bosse Ici, On Reste Ici! La grève des sanspapiers : une aventure inédite, Paris, La Découverte.

Belze C. (2011), Cartographie problématisée de l'aide à domicile en région Rhône-Alpes, Rapport de la Sécafi, commandé et financé par la CGT Rhône-Alpes, dans le cadre du programme de recherche-action « Sécurisation des parcours professionnels ».

BLANChet D., Ravalet P. (1995), « Transferts, salaire minimum et services de proximité : une maquette théorique », Revиe économique, vol. 46, n 3, pp. 573-583.

BRAVERMAn H. (1976), Travail et capitalisme monopoliste. La dégradation du travail au $X^{e}$ siècle, Paris, F. Maspero. 
Budig M., England P., Folbre N. (2002), "Wages of Virtue: The Relative Pay of Care Work", Social Problems, vol. 49, $\mathrm{n}^{\circ}$ 4, pp. 455-473.

Burnod G., Chenu A. (2001), « Employés qualifiés et non qualifiés : une proposition d'aménagement de la nomenclature des catégories socioprofessionnelles », Travail et Emploi, $\mathrm{n}^{\circ} 86$, pp. 87-105.

CAHUC P. (2001), « Pourquoi y a-t-il des différences de salaires? », Reflets et perspectives de la vie économique, vol. 40, $\mathrm{n}^{\mathrm{O}} 1-2$, pp. 13-24.

CAROli È., GaUtí́ J. (2009), Bas salaires et qualité de l'emploi : l'exception française ?, Paris, Éditions Rue d'Ulm.

CArré F., Tilly C. (2012), "A Framework for International Comparative Analysis of the Determinants of Job Quality”, in Warhurst C., Carré F., Findlay P., Tilly C. (ed.), Are Bad Jobs Inevitable? Trends, Determinants and Responses to Job Quality in the Twenty-First Century, Houndmills, Palgrave Macmillan, pp. 12-183.

CASTEL R. (1995), Les Métamorphoses de la question sociale. Une chronique du salariat, Paris, Fayard.

Cattacin S., Gianni M., Mänz M. et al. (2002), Retour au travail! Le workfare comme instrument de réforme, Fribourg, Éditions universitaires.

Centre d'analyse stratégique (2006), Besoins de main-d'œuvre et politique migratoire, Rapport, Paris, Centre d'analyse stratégique.

CAISSE NATIONALE DE L'ASSURANCE MALAdIE DES TRAVAILlEURS SALARIÉS (CNAMTS) (2017), Synthèse de la sinistralité AT-MP 2016 tous CTN. Focus sur le périmètre du CTN D. Services, commerces et industries de l'alimentation, CNAMTS, Direction des risques professionnels, en ligne : http://www.risquesprofessionnels.ameli.fr/fileadmin/user_upload/document_PDF_a_ telecharger/etudes_statistiques/livret_de_sinistralite/2016/Livret\%20Sinistralit\%C3\%A9\%20 ATMP\%20CTN\%20D\%202016\%20(2017-133-D).pdf, consulté le 24 janvier 2019.

CRESSON G. (2006), « La production familiale de soins et de santé. La prise en compte tardive et inachevée d'une participation essentielle », Recherches familiales, nº 3, pp. 6-15.

Cresson G. (dir.), Delforge S., Devetter F.-X., Lemaire D. (2011), Qualité du travail, qualité des emplois et qualité d'accueil dans les métiers de la petite enfance, Rapport de recherche pour la Caisse nationale des allocations familiales, Centre lillois d'études et de recherches sociologiques et économiques.

DAUNE-RichARD A.-M. (2003), « La qualification dans la sociologie française : en quête des femmes », in Laufer J., Marry C., Maruani M. (dir.), Le Travail du genre. Les sciences sociales du travail à l'épreuve des différences de sexe, Paris, La Découverte, Mage, pp. 138-150.

Debonneuil M. (2008), Les Services à la personne : bilan et perspectives, Document d'orientation, Paris, Inspection générale des finances.

Delphy C. (2015), Pour une théorie générale de l'exploitation : des différentes formes d'extorsion de travail aujourd'hui, Paris, Éditions Syllepse. 
DENIS J.-M. (2018), « Asseoir la représentation sur la communauté de travail. À la recherche de la communauté perdue dans la branche de la propreté », Sociologie du travail [en ligne], vol. 60, $\mathrm{n}^{\mathrm{o}} 1$ : https://journals.openedition.org/sdt/1748, consulté le 25 janvier 2019.

Devetter F.-X. (dir.), Abasabanye P., Bailly F., Barrois A., Brolis O., Chapelle K., Dussuet A., Léné A., Nirello L., Puissant E., Prouteau L. (2016), La Qualité des emplois dans la crise : comparaison des secteurs public, privé et associatif, Rapport pour la Dares.

Devetter F.-X., Dussuet A., Puissant E. (2017), « Pourquoi les aides à domicile sont-elles davantage rémunérées dans certains départements ? "Revue d'économie régionale et urbaine, $\mathrm{n}^{\mathrm{o}} 2$, pp. 239-270.

Devetter F.-X., JanY-CATRICE F. (2010), « L'invention d'un secteur et ses conséquences socioéconomiques : les politiques de soutien aux services à la personne », Politiques et management public, vol. 27, $\mathrm{n}^{\mathrm{o}} 2$, pp. 75-101.

Devetter F.-X., Jany-Catrice F., Ribault T. (2015), Les Services à la personne, nouvelle éd., Paris, La Découverte.

DevetTer F.-X., Messaoudi D. (2013), « Les aides à domicile entre flexibilité et incomplétude du rapport salarial : conséquences sur le temps de travail et les conditions d'emploi », Revue de l'Ires, $\mathrm{n}^{\mathrm{o}} 78$, pp. 51-76.

Devetter F.-X., Rousseau S. (2009), « Comment concilier service relationnel et marges réduites ? Le cas du secteur du ménage à domicile », Gestion 2000, nº 2, pp. 193-209.

Devetter F.-X., Steinauer O. (2017), L'Expérimentation de l'externalisation de l'entretien des collèges, Rapport pour le conseil départemental du Nord.

Devetter F.-X., SteInAuer O. (2018), Le Travail des aides à domicile : prescriptions et temporalités, Document de travail, Lille, Clersé.

Doeringer P. B., Piore M. J. (1971), Internal Labor Market and Manpower Analysis, Heath, Lexington Books.

Dussuet A. (1997), Logiques domestiques. Essai sur les représentations du travail domestique chez les femmes actives de milieu populaire, Paris, L'Harmattan.

Dussuet A. (2005), Travaux de femmes. Enquêtes sur les services à domicile, Paris, L'Harmattan.

Dussuet A. (2010), Femmes et services : entre public et privé, Mémoire original pour l'habilitation à diriger des recherches, Tours, Université François Rabelais.

Dussuet A. (2013), « Santé au travail et prévention des risques professionnels dans les associations d'aide à domicile », Revue de l'Ires, n 78, pp. 77-97.

Dussuet A., Nirello L., Puissant E. (2017), « De la restriction des budgets des politiques sociales à la dégradation des conditions de travail dans le secteur médico-social », Revue de l'Ires, n $^{\circ}$ 91-92, pp. 185-211.

Dussuet A., Puissant E. (2015), « Un rôle ambivalent des associations dans la reconnaissance du travail salarié. L'exemple de l'aide à domicile », Économies et sociétés, série « Socioéconomie du travail », nº 37, pp. 991-1018. 
Dwyer R. E. (2013), “The Care Economy? Gender, Economic Restructuring, and Job Polarization in the U.S. Labor Market", American Sociological Review, vol. 78, n 3, pp. 90-416.

ESTRADE M.-A. (2013), «Les emplois non pourvus : mythes et réalités », Regards croisés sur l'économie, $\mathrm{n}^{\mathrm{o}} 13$, pp. 151-167.

Fagan C., Hebson G., Unit G. (2006), Making Work Pay. Debates from a Gender Perspective. A Comparative Review of Some Recent Policy Reforms in Thirty European Countries, Luxembourg, Office for Official Publication of the European Communities.

Fagnani J., Math A. (2012), « Des assistantes maternelles mieux formées et plus qualifiées. Les parents consentiraient-ils à augmenter la rémunération ? », Politiques sociales et familiales, $\mathrm{n}^{\mathrm{o}} 109$, pp. 59-73.

FERnandez-Macias E. (2012), "Job Polarization in Europe? Changes in the Employment Structure and Job Quality, 1995-2007”, Work and Occupations, vol. 39, n 2, pp. 157-182.

Folbre N. (2001), The Invisible Heart: Economics and Family Values, New York, The New Press.

Gadrey J. (2003), Socio-économie des services, $3^{\mathrm{e}}$ éd., Paris, La Découverte.

Gadrey N., Jany-Catrice F., Pernod-Lemattre M. (2004), « Les non-qualifiés : qui sontils ? », in MÉDA D., Vennat F., Le Travail non qualifié. Permanences et paradoxes, Paris, La Découverte, pp. 166-181.

Gadrey N., Jany-Catrice F., Pernod-Lemattre M. (2009), «Employés non qualifiés : la catégorie oubliée des politiques d'égalité professionnelle », Économies et sociétés, série «Socio-économie du travail », n 30, pp. 57-86.

Gautié J., Margolis D. (2009), «L'impact de la politique publique sur le marché du travail à bas salaire : offre, demande et qualité de l'emploi », Économie et statistique, n 429-430, pp. 3-19.

Gollac M., Volkoff S. (2006), «La perception subjective du travail : rôle des identités de genre et des conditions d'emploi (quelques éléments d'analyse statistique) », Document de travail, $\mathrm{n}^{\circ} 69$, Centre d'études de l'emploi.

Gordon D. M., Edwards R., ReIch M. (1982), Segmented Work, Divided Workers: The Historical Transformation of Labor in the United States, Cambridge, Cambridge University Press.

Grimshaw D., Cartwright J., Keizer A., Rubery J. (2014), Coming Clean: Procurement and Contract Practices in Commercial Cleaning, Equalities and Human Rights Commission, Manchester, EHRC.

Guilbert M. (1966), Les Fonctions des femmes dans l'industrie, Paris, Mouton et C $\mathrm{C}^{\mathrm{ie}}$.

HaAkkestad H., Fridberg J. H. (2017), "Deskilling Revisited: Labour Migration, Neo-Taylorism and the Degradation of Craft Work in the Norwegian Construction Industry", Economic and Industrial Democracy [en ligne] : https://journals.sagepub.com/ doi/10.1177/0143831X17735671, consulté le 25 janvier 2019. 
Hély M. (2009), Les Métamorphoses du monde associatif, Paris, Presses universitaires de France.

Hipp L., Bernhardt J., Allmendinger J. (2015), "Institutions and the Prevalence of Nonstandard Employment”, Socio-Economic Review, vol. 13, n² 2, pp. 351-377.

Holley S., Rainnie A. (2012), "Who Cleans Up? The Declining Earnings Position of Cleaners in Australia”, The Economic and Labour Relations Review, vol. 23, n ${ }^{\circ}$ 1, pp. 143-160.

Iвоs C. (2013), « Quand la garde d'enfants se mondialise », Plein droit, n ${ }^{\circ}$ 96, pp. 7-10.

JAEHRLING K., MÉHaUt P. (2013), “'Varieties of Institutional Avoidance’: Employers’ Strategies in Low-Waged Service Sector Occupations in France and Germany", Socio-Economic Review, vol. $11, \mathrm{n}^{\circ} 4$, pp. 687-710.

JANY-CATRICE F. (2010), « La construction sociale du "secteur" des services à la personne : une banalisation programmée ? », Sociologie du travail, vol. 52, nº 4, pp. 521-537.

JoLly C. (2015), «La polarisation des emplois : une réalité américaine plus qu' européenne ? », Document de travail, $\mathrm{n}^{\mathrm{o}}$ 2015-04, France Stratégie.

Jounin N. (2009), Chantier interdit au public. Enquête parmi les travailleurs du bâtiment, Paris, La Découverte.

Kalleberg A. (2011), Good Jobs, Bad Jobs, New York, Russel Sage Foundation.

Kulanthaivelu É. (2018), «Les services à la personne en 2016. L'activité se stabilise », Dares résultats, $\mathrm{n}^{\circ} 017$.

Lamotte B., Puissant E. (2010), Recherche-action « La précarité dans l'emploi, nouvelles formes d'emploi et de travail dans l'aide à domicile », Contribution au rapport final du programme.

LAmotTe B., Puissant E. (2013), Recherche-action « Sécurisation des parcours professionnels et dialogue social territorial dans le secteur de l'aide à domicile », Contribution au rapport final du programme.

LE Roy A., Puissant E. (2016), « Évolution des référentiels politiques dans l'aide à domicile », RECMA, n ${ }^{\circ} 342$, pp. 69-84.

LEFEBVRE M. (2012), Qualité(s) de l'emploi dans les services à la personne : entre régulations publiques et professionnelles, Thèse de doctorat en sciences économiques, Université Lille 1.

Marglin S. (1974), "What bosses do?", Review of Radical Political Economics, vol. 6, n², pp. 60-112.

MARSDEN D. (1999), A Theory of Employment Systems, Oxford, Oxford University Press.

Martin-HuAn J. (1997), La Longue Marche des domestiques en France : du XIXé siècle à nos jours, Nantes, Opéra éditions.

Maruani M., Nicole-Drancourt C. (1989), Au labeur des dames : métiers masculins, emplois féminins, Paris, Syros-Alternatives.

MARX K. (1867), Le Capital. Euvres I, Paris, Gallimard. 
MÉda D., PÉRIVIER H. (2007), Le Deuxième Âge de l'émancipation. La société, les femmes et l'emploi, Paris, Seuil.

Méda D., Vennat F. (2004), Le Travail non qualifié. Permanences et paradoxes, Paris, La Découverte.

Messing K., Chatigny C., Courville J. (1998), “'Light' and 'Heavy' Work in the Housekeeping Service of a Hospital”, Applied Ergonomics, vol. 29, n 6, pp. 451-459.

Messing K., Doniol-Shaw G., HaËntJEns C. (1993), "Sugar and Spice and Everything Nice: Health Effects of the Sexual Division of Labor among Train Cleaners", International Journal of Health Services, vol. 23, n ${ }^{\circ} 1$, pp. 133-146.

Nirello L. (2015), La Construction problématique de la relation d'emploi dans l'ESS : les Ehpad, entre régulations publiques et régulations d'entreprise, Thèse de doctorat en sciences économiques, Université de Nantes.

Nkouatchet R. N. (2005), « La précarité de l'emploi au service de la prospérité du fast-food », Sociologie du travail, vol. 47, $\mathrm{n}^{\circ} 4$, pp. 470-484.

Oesch D., Menes J. (2011), "Upgrading or Polarization? Occupational Change in Britain, Germany, Spain and Switzerland, 1990-2008”, Socio-Economic Review, vol. 9, n 3, pp. 503-531.

Perraudin C., Thèvenot N., Tinel B., Valentin J. (2006), « Sous-traitance dans l'industrie et ineffectivité du droit du travail : une analyse économique », Économie et institutions, $\mathrm{n}^{\circ} 9$, pp. 35-55.

PuISSANT E. (2010), La Relation associative d'aide à domicile : spécificités, remises en cause, résistances, Thèse de doctorat en sciences économiques, Université de Grenoble.

Puissant E. (2011), «Le rôle ambivalent des associations d'aide à domicile dans la professionnalisation des emplois et des salariées », Formation emploi, n ${ }^{\circ} 115$, pp. 37-50.

Rose J. (2012), Qu'est-ce que le travail non qualifié ?, Paris, La Dispute.

Roux N. (2018), « De l'emploi stable au travail insoutenable. Trajectoires d'ouvrières agricoles en groupement d'employeurs », Document de travail, n $^{\circ} 196$, Centre d'études de l'emploi et du travail.

THIÉRUS L. (2014), « Les services à la personne en 2012. Baisse de l'activité, sauf dans les entreprises prestataires », Dares analyses, $\mathrm{n}^{\circ} 038$.

TIRMARCHE-ISSEMANN A. (2011), L'Institutionnalisation de la fonction d'assistante maternelle. La reconfiguration de l'économie domestique, Thèse de doctorat en sociologie, Université de Strasbourg.

Trabut L. (2014), «Aides à domicile : la formation améliore-t-elle l'emploi ? », Formation emploi, $\mathrm{n}^{\mathrm{o}} 127$, pp. 71-90.

VATAN S. (2014), La Tarification des services d'aide à domicile : une analyse institutionnaliste par le rôle paramétrique du prix, Thèse de doctorat en sciences économiques, Université Lille 1.

VOZARI A.-S. (2014), « Recruter de “bonnes” assistantes maternelles. La sélection à l'entrée d'un emploi féminin non qualifié », Sociétés contemporaines, $\mathrm{n}^{\circ}$ 95, pp. 29-54. 
Western B., HeAly K. (1999), "Explaining the OECD Wage Slowdown Recession or Labour Decline?”, European Sociological Review, vol. 15, n 3, pp. 233-249.

ZaJdela H. (2009), « Comment et pourquoi activer les inactifs ? ", Travail et Emploi, $\mathrm{n}^{\circ} 118$, pp. 69-76.

Zock J. P. (2005), "World at work: Cleaners", Occupational and Environmental Medicine, vol. $62, \mathrm{n}^{\mathrm{o}} 8$, pp. 581-584. 


\section{ANNEXe - PrincipauX MatériauX EMPIRIQUeS}

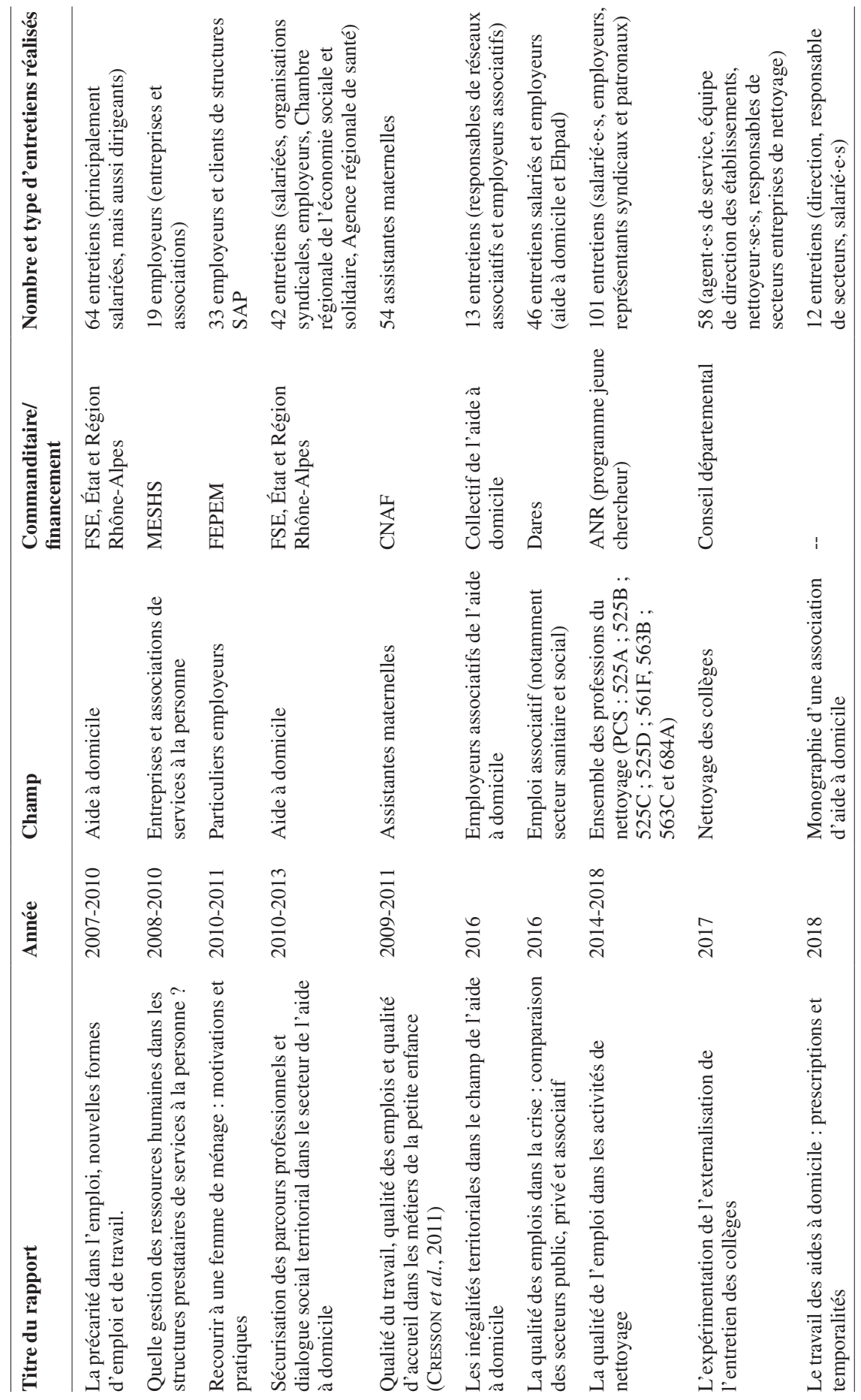


